\title{
Voting Rules in Sequential Search by Committees: Theory and Experiments
}

\author{
Vincent Mak \\ Cambridge Judge Business School, University of Cambridge, \\ Trumpington Street, Cambridge CB2 1AG, United Kingdom \\ v.mak@jbs.cam.ac.uk \\ Darryl A. Seale \\ Lee Business School, University of Nevada, Las Vegas, \\ 4505 Maryland Parkway - Box 456009 Las Vegas, NV 89154-6009 \\ darryl.seale@unlv.edu \\ Amnon Rapoport* \\ A. Gary Anderson Graduate School of Management, \\ Anderson Hall, University of California, Riverside, CA 92521 \\ amnonr@ucr.edu \\ Eyran J. Gisches \\ Eller College of Management, University of Arizona, \\ 1130 E. Helen St. McClelland Hall, Tucson, AZ 85721 \\ eyran@email.arizona.edu
}

May 29, 2018

* Corresponding author 


\title{
Voting Rules in Sequential Search by Committees: Theory and Experiments
}

\begin{abstract}
We propose a committee extension of the individual sequential search model called the "secretary problem," where collective decisions on when to stop the search are reached via a pre-specified voting rule. We offer a game-theoretic analysis of our model, and then report two experiments on three-person committees with either uncorrelated or perfectly correlated preferences under three different voting rules, followed by a third experiment on single decision makers. Relative to equilibrium predictions, committees with uncorrelated preferences over-searched under minority and majority voting rules, but otherwise undersearched or approximated equilibrium play. Individually, committee members were often less strategic when their preferences were uncorrelated than when they were perfectly correlated. Collectively, committees' decisions were more strategic than single decision makers' only under the unanimity rule, though still not significantly better in terms of the decision makers' welfare. Finally, across our experiments with committee search, the unanimity rule always optimized committee welfare.
\end{abstract}

Keywords: Committee sequential search; Voting rules; Secretary problem; Experiments 


\section{Introduction}

Sequential search is a dynamic decision process in which alternatives are inspected one at a time to be either accepted or rejected, and the process terminates once an alternative is accepted. A fruitful theoretical and empirical literature on individual sequential search has emerged in the past decades, particularly with applications to individual job search (see, e.g., the reviews in Lippman and McCall 1976, and Rogerson, Shimer, and Wright 2005). In the present study, we investigate committee sequential search, which has rarely been examined in previous experimental research. Common managerial decisions such as hiring personnel, renting office space, and selecting investment projects, are frequently made by committees whose members evaluate alternatives one at a time in the fashion of a sequential search. Common household decisions, such as sequentially searching for accommodation while travelling on the road, are often made by "committees" that include the husband, wife, and one or more family members. These processes involve not only dynamic individual decisions, as in individual sequential search problems, but also dynamic strategic interactions among committee members with alternative mechanisms for resolving disagreements, which bring in new elements of analytical and behavioral interests with managerial implications.

Sequential search by committees has two crucial characteristics. The first is that committee members frequently have diverse preferences over the alternatives. For example, when the board of directors of a firm evaluates its applicants for a senior executive position, one board member might consider internal communications skills to be of utmost concern, the second may be more concerned with finance abilities, and the third may consider market savviness to be most important. As members of the board consider applicants for the position, one at a time, they may hold different evaluations over the sequentially interviewed candidates. While committee members' rankings over applicants should probably be aligned to some extent, their preferences are typically only partially correlated. Moreover, if senior executives are extremely high in demand in the job market, while interviews are spaced relatively far apart because of logistical and administrative issues, then the board will have to make a prompt accept/reject decision to every interviewed candidate. Effectively every rejected candidate will have found employment quickly and cannot be recalled, highlighting the importance of every single committee decision. 
The second characteristic in committee search is the amount or quality of the information available to the decision makers (DMs). The two major information structures that have been discussed in the literature include the full-information case (e.g., Albrecht, Anderson, and Vroman 2010, Hizen, Kawata, and Sasaki 2013, Inukai, Kawata, and Sasaki 2017), where the entire distribution of the quality of the alternatives is assumed to be fully known and accurately specified before the observation and selection process commences. As Palley and Kremer (2014) have noted, the assumptions behind the full-information case seem restrictive in many contexts. These include, for example, the case where the search context is new and unfamiliar to the DMs, such as a board of entrepreneurs recruiting an executive for a new venture in an unfamiliar industry. In those cases, each DM has scant experience in assigning a value to any one alternative. Instead, she may only be able to make ordinal preference comparisons over the inspected alternatives. Those situations pertain to the no-information or partial-information case (e.g., Abdelaziz and Krichen 2007, Mak, Rapoport, and Seale 2014), where only the rank of each alternative, once it is inspected, may be determined during the search relative to the ranks of all the alternatives that have been inspected and subsequently rejected.

The present study focuses on the no-information case. As in the individual search scenario, it has been modelled by multiple variants of the "secretary problem" (SP; see e.g., Ferguson 1989). Here, we propose a committee extension of the secretary problem with a model that accommodates any level of partially correlated preferences among committee members. We report a game-theoretic analysis of our model followed by two experiments with three-person committees, one with uncorrelated and the other with perfectly correlated preferences, as well as a third experiment with single DMs. Three different voting rules, which aggregate individual decisions over an alternative into a committee decision, are tested in our experiments.

\subsection{Research Objectives}

Our study investigates three major research objectives:

(1) What kind of decision improvement effort will be desirable to committee decision making? 
(2) Does a committee with strongly aligned or perfectly correlated preferences necessarily make better decisions than an individual?

(3) What might be the best voting rule for aggregating individual decisions into the collective committee decision?

Our analysis is based on strategic benchmarks that optimize the decisions of committee members (with respect to their self-interest) in response to each other. We employ game-theoretic equilibrium analysis to arrive at these predictions. Equilibrium strategies are premised on every committee member making optimal decisions in response to each other and pre-empting every scenario of future conflict, as with backward induction in a sequential decision game. A crucial empirical question, which calls for a behavioral approach, is whether humans could approach such benchmark even upon considerable learning. Accordingly, our experiments examine whether, upon repeated interactions, laboratory subjects approach our equilibrium benchmarks. The benchmarks should thus be considered a prescriptive yardstick rather than a descriptive model; its prescriptive value is further highlighted by several reasons. First, when preferences are weakly aligned or uncorrelated, equilibrium forms the only self-consistent set of "point" predictions that is stable against self-interested deviations. Secondly, when preferences are strongly aligned or perfectly correlated, equilibrium and welfare-maximizing benchmarks coincide and are highly desirable. Lastly, in a set of additional analysis on our experimental data, we estimated the empirical best response strategies with respect to the experimental population and tested observed decisions against them. The outcomes of this analysis are consistent with our conclusions on how sophistication of our subjects changes with preference correlation, voting rule, or committee size.

Our first research objective is related to whether and how individuals might systematically deviate from the strategic benchmarks. The directions of the deviations might inform us about what decision improvement effort would be desirable. We find that experimental subjects with uncorrelated preferences over-searched with respect to equilibrium predictions under the minority and majority voting rules; otherwise, they under-searched or approximated equilibrium play. Individually, committee members were often less strategic when their preferences were uncorrelated than when they were perfectly correlated. 
Our second research objective is to determine whether a committee with aligned preferences necessarily makes better decisions than an individual in a similar task. We find that committee members' individual decisions are less strategic than single DMs when preferences are uncorrelated as well as perfectly correlated. At a collective level, committees' decisions were closer to benchmark predictions on optimal search (i.e., "smarter") than single decision makers' decisions when the threshold number of votes to stop the search is sufficiently high - though still without a significant welfare improvement. That is, the committee's collective decisions might be "smarter" than the individual's in those cases but not significantly better.

Our third research objective is related to how we can design the committee decision process to improve outcomes. We find that when preferences were uncorrelated, experimental committees made the best decisions (in terms of committee welfare) under the unanimity voting rule - which is consistent with theoretical predictions. On the other hand, when preferences were perfectly correlated, in theory the voting rules should all have yielded the same level of welfare; but in the corresponding experiment, compared with the minority voting rule, the majority and unanimity rules seemed to function better as a check against the main tendency of individuals to under-search, and achieved superior committee welfare. As a rule, across our two experiments with committee search, the unanimity rule always optimized committee welfare.

We begin Section 2 by briefly reviewing the relevant literature. Sections 3 and 4 present the analytical specification of our model, and outline the procedures by which we compute a set of equilibrium strategies for the model. Sections 5 to 8 describe the three experiments and their results. Section 9 discusses overall methodological issues. Section 10 concludes with a discussion of our findings, managerial implications, and directions for future research.

\section{Literature Review}

In this section, we offer a brief overview of a set of studies that are related to the present research. Table 1 presents a summary of our discussion, together with the main features of the present study.

\subsection{The Secretary Problem (SP) as a Theoretical Paradigm}


The search model in our study is a committee extension of the secretary problem (SP). Under all variants of the SP, the decision task involves determining whether an alternative is to be chosen based on its relative rank among the alternatives inspected so far. In the classical version of the SP (e.g., Lindley 1961), the objective of the DM is to maximize the probability of choosing the best among all the alternatives that can potentially be inspected. Therefore, the classical SP is referred to as the best choice SP (BCSP). There have been many extensions of the BCSP (see e.g., Freeman 1983, Samuels 1991, Stein et al. 2003, and Bearden, Rapoport, and Murphy 2006). In a second major variant of the problem, Chow et al. (1964) consider the problem of finding an optimal stopping rule for choosing an alternative out of a finite sequence that minimizes the expected value of the absolute rank of the selected alternative. This class of problems have been referred to as expected rank SPs (ERSP), and form the basis of the present study. Theoretical extensions of the SP to committee search are more recent (see e.g., Sakaguchi and Mazalov 2004, Abdelaziz and Krichen 2007). They predominantly focus on two-person committees. There have been scarcely any attempts to extend SP to multiple-person committee search.

\subsection{Individual Sequential Search Experiments}

There is a rich literature of experimental studies on individual full-information search that largely stems from investigations of job-search decisions (Schotter and Braunstein 1981, Braunstein and Schotter 1982, Cox and Oaxaca 1989, Harrison and Morgan 1990, Brown et al. 2011). The experimental literature using variants of the BCSP to model no-information search has begun more recently, and is reviewed by Bearden and Rapoport (2005), Bearden, Murphy, and Rapoport (2005), and Bearden et al. (2006). Nearly all the previous studies considered a single DM and found under-search behavior relative to the optimal strategy. Focused attempts to understand sequential search decisions by means of heuristics and other behavioral factors include Seale and Rapoport (1997, 2000), Sonnemans (1998), Zwick, Rapoport, Lo, and Muthkrishnan (2003), Schunk (2009), and Schunk and Winter (2009). Palley and Kremer (2014) offer a useful comparison between cases with two different levels of information in an experimental study on individual sequential search, and discuss their respective merits in modelling real-world search decisions.

\subsection{Committee Sequential Search Experiments; Contributions of the Present Study}


To the best of our knowledge, there are only a few experimental studies on sequential search by committees, including Hizen et al. (2013) - with Inukai et al. (2017) a recent extension - and Mak et al. (2014). Our present work and these existing studies might be perceived as complementary in building a nascent stream of experimental research on committee sequential search.

Hizen et al. (2013) investigated three-person committee search as in the present study; Inukai et al. (2017) experimented on the same framework but with ex ante heterogeneity among the decision makers. In both studies, the setup is based on the infinite horizon, full-information search model of Albrecht et al. (2010), which is drastically different from the present study with finite horizon and no-information search. The focus of their line of work was on the comparison of search behavior between committee and singleDM search, as well as the study of committee search contingent on the number of votes required to stop it. Their general findings seem to be that DMs are more ready to accept alternatives in committee search than in single-DM search, suggesting a prevalence of Type I deviations (see Table 4 and related discussion), and more under-search among committees than among single DMs. In comparison, our findings are more nuanced. Fundamentally, our finite-horizon setup brings about a stronger need for strategic concerns than infinite horizon search: unlike infinite horizon search, the DMs in a finite horizon search ought to factor in the possibility of exhausting all or most of the available alternatives without any agreement and end up having to compromise with inferior final alternatives.

Mak et al. (2014) studied two-person committee search based on Sakaguchi and Mazalov (2004)'s model. As in the present study, Mak et al. (2014) used a no-information setup with finite horizon that follows the SP paradigm. However, their independent variables were committee members' relative power over the final outcome (the probability that in case of disagreement a tie would be broken in favor of one member rather than the other) and information flow (whether the committee members were informed about each other's preferences over the inspected alternatives); over-searching was prevalent in their experiment although its extent could be mitigated by increased information flow.

Neither of the independent variables in Mak et al. (2014) is the focus of the present study. Rather, the present study focuses on voting rules and preference correlations in three-person committee search with 
additional benchmarking results from a single-DM search experiment. The committee members in the present study have homogeneous power; the manipulation of voting rule is a requirement, given such homogeneity, on how members' decisions are aggregated into the committee's decision. The focus on voting rules highlights a dimension of the strategic complexity of committee search that has been unexplored by Mak et al. (2014); our findings that, as a rule, the unanimity rule optimized committee welfare across experiments, are categorically distinctive from the previous study's findings. Mak et al. (2014) found over-search behavior and non-significant deviations from equilibrium payoffs across all conditions. In contrast, the present study uncovers over-search (minority and majority rules in Experiment 1), under-search (unanimity rule in Experiment 1, minority and majority rules in Experiment 2 and Experiment 3), and approximately equilibrium search (unanimity rule in Experiment 2) with significant deviations from equilibrium payoffs.

Our findings caution against simplistic assumptions about the quality of committee decision making over individual decision making. This invites a brief overview of related findings in previous literature. Overall, previous literature suggests that groups make better and more strategic decisions than individuals in individual and interactive decision tasks (see, e.g., Charness and Sutter 2012 and the studies discussed therein; and Sutter, Czermak, and Feri 2013). But the experimental decision tasks in those earlier studies were typically much simpler than the present one. In our study, on the question of whether groups make better decisions than individuals, we find that the answer depends on the voting rule - or, more broadly, on how individual decisions are aggregated into the group's decision.

\section{The Committee Search Model}

\subsection{Individual Preferences}

A committee of $m$ members $(m \geq 2)$ consider a set of $n$ alternatives that are presented to them sequentially, one at a time, in a random order. As in the classical SP, we assume that every member can rank-order the $n$ alternatives in terms of their desirability $(1=$ best, $2=$ second best, etc. $)$ with no ties, so that when the committee is inspecting the $j$-th alternative every member $i$ (indexed by $i=1,2, \ldots m$ ) has a well-defined 
Table 1 Summary of the Reviewed Literature in Comparison with the Present Study

\begin{tabular}{|c|c|c|}
\hline Reviewed studies & Features & Highlighted empirical findings \\
\hline \multicolumn{3}{|c|}{ The Secretary Problem (SP) as a theoretical paradigm } \\
\hline $\begin{array}{l}\text { Lindley (1961), Freeman (1983), } \\
\text { Samuels (1991), Stein et al. (2003), } \\
\text { Bearden et al. (2006) }\end{array}$ & $\begin{array}{l}\text { Classical best choice } S P \\
(B C S P) \text { models }\end{array}$ & \multirow{3}{*}{ Not applicable } \\
\hline Chow et al. (1964) & $\begin{array}{l}\text { Expected rank } S P(E R S P) \\
\text { model }\end{array}$ & \\
\hline $\begin{array}{l}\text { Sakaguchi and Mazalov (2004), } \\
\text { Abdelaziz and Krichen (2007) }\end{array}$ & $\begin{array}{l}\text { Committee search extensions; } \\
\text { predominantly two-person } \\
\text { committees }\end{array}$ & \\
\hline
\end{tabular}

Individual sequential search experiments

Schotter and Branustein (1981),

Braunstein and Schotter (1982),

Cox and Oaxaca (1989),

Harrison and Morgan (1990),

Full-information search,

Brown et al. (2011)

Bearden and Rapoport (2005),

Bearden et al. (2005), No-information search Largely consistent findings of

Bearden et al. (2006)

Seale and Rapoport (1997, 2000), Full-/no-information search

Sonnemans (1998), Zwick et al. (2003), with focused examination on

Schunk (2009),

heuristics and other

Schunk and Winter (2009)

behavioral factors

Palley and Kremer (2014)

Comparisons of full-vs. partial-information search

Committee sequential search experiments

Three-person committees, full- More under-search among information search committees than among single DMs

Two-person committees, noinformation search; examines committee members' relative power, and information flow

Quality of group decision making

Groups vs. individual comparisons in individual decision tasks and strategic games

information search

\section{Prevalent over-search}

Groups make better and more strategic decisions than individuals under-search across studies
Hizen et al. (2013), Inukai et al. (2017)

\begin{tabular}{l} 
Mak et al. (2014) \\
\hline \\
Charness and Sutter (2012), \\
Sutter et al. (2013)
\end{tabular}

Sutter et al. (2013)

The present study Under-, over-, and approximately
optimal search depending on
preference correlation and voting
rule; depending on voting rule,
single DMs could make better
decisions with less under-search
than committees; across
experiments, unanimity rule
optimized committee welfare

relative rank $R_{i, j}$ for that alternative with respect to all previously inspected alternatives. Members' 
correlated. For the $j$-th alternative $(j>1)$, the relative ranks $R_{i, j} \mathrm{~s}$ of the committee members are generated according to the following two-step stochastic process:

(1) The first step of the process is a random draw of an integer $\bar{R}_{j}$ from the discrete uniform distribution over $\{1,2, \ldots j\}$, i.e., $P\left(\bar{R}_{j}=R\right)=1 / j$ for $R=1,2, \ldots j$;

(2) Given the realized $\bar{R}_{j}$, the second step of the process is to generate the sequence $\left\{R_{1, j}, R_{2, j}, \ldots R_{i, j}, \ldots\right.$ $\left.R_{m, j}\right\}$ such that each element in the sequence is a random variable that is independently drawn from the following distribution:

$$
\begin{aligned}
& P\left(R_{i, j}=R \mid \bar{R}_{j}=R\right)=\frac{1+(j-1) \sqrt{\mu}}{j}, \text { and } \\
& P\left(R_{i, j}=R^{\prime} \mid \bar{R}_{j}=R\right)=\frac{1-\sqrt{\mu}}{j} \text { when } j>1 \text { and } R^{\prime} \in\{1,2, \ldots j\} \backslash\{R\},
\end{aligned}
$$

where $\mu$ is a real-number parameter of the model defined over the range $0 \leq \mu \leq 1$. Thus $R_{i, j}=\bar{R}_{j}$ with probability $[1+(j-1) \sqrt{\mu}] / j$, and is equal to any value other than $\bar{R}_{j}$ with probability $(1-\sqrt{\mu}) / j$; the latter probability is equal for all those $j-1$ possible values. We may interpret $\bar{R}_{j}$ as, probabilistically, representing a "modal preference" among committee members regarding the $j$-th alternative relative to all previously inspected alternatives. In Online Appendix A.1 we show that when $j>1$ :

$$
\operatorname{Corr}\left(R_{i, j}, R_{i^{\prime}, j}\right)=\mu \text { for any two different committee members } i \text { and } i^{\prime} \text {. }
$$

Hence, $\mu$ measures the correlation of preference vectors among committee members over the $n$ alternatives.

\subsection{Individual Decisions}

The individual decision task involves each committee member $i$ observing his/her own $R_{i, 1}, R_{i, 2}, \ldots$ in sequence. At the inspection of each alternative, member $i$ decides whether to accept the alternative or reject it with no recall possibility (compare, e.g., the decisions in the partial-information model of Palley and 
Kremer 2014, as well as previous SP models). Each committee member submits this (binary) decision by casting a vote without knowing the other committee members' relative ranks or individual decisions.

\subsection{Aggregation of Individual Decisions into Committee Decision}

The accept/reject decisions of the committee members regarding the currently inspected alternative are aggregated into the committee's decision via a pre-determined and commonly known voting rule. We focus on a general class of voting rules that assume homogenous members, namely, that the committee accepts the current alternative if a threshold number of $q$ members accept it, where $q$ can be $1,2,3 \ldots m$. It is useful to define the following special cases for subsequent discussion:

- Minority voting rule $(q \leq m / 2)$ - the threshold is not more than half of the committee.

- Majority voting rule $(q=(m / 2)+1$ if $m$ is even; $q=(m+1) / 2$ if $m$ is odd $)$ - the threshold is essentially half of the committee.

- Unanimity voting rule $(q=m)$ - the threshold is the full committee.

3.3.1. Discussion. Although the committee is making its decision over $n$ alternatives, the decision is decomposed into a sequence of binary accept/reject decisions, each occurring at a decision stage when an alternative is inspected. At each decision stage, the committee decision is also a binary one that is aggregated over the $m$ individual decisions. From this perspective, our voting rule framework subsumes several common voting procedures. For example, the Borda count method (e.g., Nurmi 1987) as applied to our context would involve each committee member ranking between accept and reject, and then counting whether there are more accepts than rejects, or vice versa, in order to arrive at a committee decision. Therefore, it corresponds to majority voting in our model. The Condorcet method (e.g., Black 1958, Nurmi 1987) would involve committee members conducting majority voting over every pair of possible decisions at each decision stage, which here is trivially only the pair of accept/reject decisions; thus, the Condorcet method also corresponds to majority voting in our model.

\subsection{Individual Payoffs from the Search}

Once the committee accepts an alternative, the search is terminated; if the committee rejects the first $n-1$ alternatives, then the last ( $n$-th) alternative is accepted by default. Once the search is stopped, the absolute 
ranks of every member over all the $n$ alternatives are revealed. If the $j$-th alternative is accepted, member $i$ earns a utility of $u_{i, j}{ }^{n}=B-C \cdot A_{i, j}^{n}$, where $B$ and $C$ are constants with $B \geq 0, C>0$, and $A_{i, j}^{n}$ is the absolute rank of the $j$-th alternative to $i$ among the $n$ alternatives. Therefore, an expected utility maximizing committee member will seek to minimize his/her own expected absolute rank of the accepted alternative.

\section{Equilibrium Strategies}

Here we outline the algorithmic procedure for computing equilibrium strategies for our model that are based on game-theoretic equilibrium concepts - by which every member responds optimally to other members in every possible realization of the game. Our approach follows backward induction procedures for solving sequential games. It is recursive, beginning with how decisions will be made if the game reaches the final ( $n$-th) alternative, and then working backwards to determine mutual best responses at every decision stage.

Let $V_{i, j, k .}=E\left(A_{i, j}^{n} \mid R_{i, j}=k\right)$ be the expected absolute rank or "value" of the $j$-th alternative with relative rank $k$ for committee member $i$. As noted previously, regardless of the preference correlations among committee members, the marginal distribution of a committee member's relative rank is the same discrete uniform distribution as in the single-DM search problem. Hence, the formula for $V_{i, j, k .}$ is also the same as in the single-DM problem, namely (see, e.g., Chow et al. 1964):

$$
V_{i, j, k}=\frac{n+1}{j+1} \cdot k
$$

The committee member prefers stopping the search if $V_{i, j, k}$ is not higher than $\bar{U}_{i, j}$, the expected absolute rank or "value" to be obtained by the member if the $j$-th alternative is rejected by the committee, and the search proceeds to the $(j+1)$-th alternative. $\bar{U}_{i, j}$ is only defined for $j=1,2, \ldots n$-1 because the $n$-th alternative must be accepted by the committee. $\bar{U}_{i, j}$ depends on the members' decision strategies in future decision stages of the search whereas $V_{i, j, k}$ does not. Specifically:

$$
\bar{U}_{i, j}=\frac{1}{j+1} \sum_{d=1}^{j+1} U_{i, j+1, d} \text { for } j=1,2, \ldots n-1,
$$


where $U_{i, j+1, d}$ is the expected absolute rank to be obtained by $i$ conditioned on: (i) the search having proceeded to the $j+1$-th alternative (so that the committee has rejected alternatives $1,2, \ldots j$ ), (ii) $R_{i, j+1}=d$, and (iii) all the committee members following the equilibrium strategy. Formally:

$$
U_{i, j+1, d}=\sum_{j^{\prime}=j+1}^{n} P\left(\text { group accept } j^{\prime} \mid R_{i, j+1}=d\right) \times E\left(A_{j^{\prime}}^{n} \mid \text { group accept } j^{\prime} \text { and } R_{i, j+1}=d\right) \text {, }
$$

conditioned further on the search having proceeded to the $j+1$-th alternative, and all committee members following the equilibrium strategy.

For any possible $j$ and $k, U_{i, j, k}$ can be computed recursively. It is related to the probability that the $j$-th alternative with relative rank $k$ to committee member $i$ is accepted by the committee under the equilibrium strategy. Denoting this probability by $p_{i, j, k}$, we have:

$$
U_{i, j, k}=p_{i, j, k} V_{i, j, k}+\left(1-p_{i, j, k}\right) \bar{U}_{i, j} \text { for } j=1,2 \ldots n-1, \text { and } U_{i, n, k}=V_{i, n, k}=k
$$

The term $p_{i, j, k}$ is related to the threshold relative rank $c_{j}^{*}$ by which a committee member accepts the $j$-th alternative under the equilibrium strategy. Formally:

$$
c_{j}^{*}=\max \left\{0, k: V_{i, j, k} \leq \bar{U}_{i, j}\right\}
$$

Note that:

(a) By symmetry, $c_{j}^{*}$ is the same for all committee members; hence, it does not have the $i$ subscript;

(b) If the inequality on the right-hand side of (4) cannot be satisfied by any $k$, then $c_{j}^{*}=0$;

(c) The optimal decision as expressed in (4) is a dominant strategy. That is, the decision yields the highest expected payoff to member $i$ regardless of other members' decisions over the current (i.e., $j$-th) alternative. Other members' strategies only enter equation (4) via $\bar{U}_{i, j}$, i.e., in terms of what they would do in hypothetical scenarios regarding future alternatives, if the search continues;

(d) Equation (4) adopts the tie-breaking rule that if the value of accepting the current alternative is equal to the value of continuing search, then the committee member will strictly prefer accepting the current alternative. In other words, we assume that if accepting and rejecting the current alternative yield the same expected payoff, then the DM will accept the current alternative. This assumption may be justified if the 
committee members have a very small, and otherwise negligible, time preference over early resolution of uncertainty. In our three-person committee calculation (see the next subsection) with $n=40$, tie-breaking is needed in only a single case, with the (n-1)-th alternative, when a committee member faces an alternative with relative rank 20 (and expected absolute rank 20.5), which ties with continuing search. The tie-breaking rule makes no significant differences in the threshold ranks.

Given the value of $c_{j}^{*}$, we can compute $p_{i, j, k}$. Recall that the voting rule requires at least $q$ votes to stop the search. Thus, formally, $p_{i, j, k}$ is the probability of at least $q$ committee members' relative ranks being less than or equal to $c_{j}^{*}$, conditioned on the fact that one particular member $i$ has $R_{i, j}=k$. Then:

$p_{i, j, k}=\left\{\begin{array}{l}P\left(R_{r, j} \leq c_{j} * \text { for at least } q \text { group members among } r=1,2 \ldots i-1, i+1 \ldots \mid R_{i, j}=k\right) \text { if } k>c_{j} * \\ P\left(R_{r, j} \leq c_{j} * \text { for at least } q-1 \text { group members among } r=1,2 \ldots i-1, i+1 \ldots \mid R_{i, j}=k\right) \text { if } k \leq c_{j} * .\end{array}\right.$

The top row in the right-hand side of Eq. (5) corresponds to the case when $i$ rejects the alternative; the bottom row when $i$ accepts the alternative. The values of $p_{i, j, k}$, given the model parameters, can be computed from the two-step stochastic process for the relative ranks introduced earlier. For example, when $\mu=0$, so that the $R_{i, j}$ s are uncorrelated among the $m$ committee members:

$$
p_{i, j, k}=\left\{\begin{array}{l}
\sum_{r=q}^{m-1}\left(\begin{array}{c}
m-1 \\
r
\end{array}\right) \pi_{j}^{r}\left(1-\pi_{j}\right)^{m-1-r} \text { if } k>c_{j}{ }^{*} \\
\sum_{r=q-1}^{m-1}\left(\begin{array}{c}
m-1 \\
r
\end{array}\right) \pi_{j}^{r}\left(1-\pi_{j}\right)^{m-1-r} \text { if } k \leq c_{j}^{*},
\end{array}\right.
$$

where $\pi_{j}=c_{j} * / j$. When $\mu=1$, so that the $R_{i, j}$ s are perfectly correlated among the committee members:

$$
p_{i, j, k}=\left\{\begin{array}{l}
0 \text { if } k>c_{j}^{*} \\
1 \text { if } k \leq c_{j}^{*}
\end{array}\right.
$$

Equations (1) to (5) jointly define a recursive routine for computing the equilibrium strategies for our model. By construction, the equilibrium strategies are unique and strict (i.e., the equilibrium strategy is the unique best response at every decision stage of the game), apart from the tie-breaking flexibility discussed above.

\subsection{Discussion}


For expositional simplicity, and out of logistical and resource concerns for experimental investigations of the model, henceforth we focus on a minimal committee size that still offers the possibilities of minority, majority, and unanimity voting rules. This implies a focus on three-person committees $(m=3)$. Table 2 lists the complete threshold ranks under equilibrium strategies for each of the three voting rules when $n=40, m$ $=3$, and separately for $\mu=0$ (uncorrelated preferences) and $\mu=1$ (perfectly correlated preferences). (Recall that $\mu$ is the constant pairwise correlation of relative ranks between any two committee members for any alternative in our model). For instance, assume that the committee members have uncorrelated preferences, while the committee has rejected the first five alternatives and is presently inspecting the $6^{\text {th }}$ alternative. Table 2 shows that in equilibrium a member should accept the current alternative if its relative rank is 3 or less, 2 or less, or 1, under the minority, majority, and unanimity voting rule, respectively. Note that when $\mu=1$, the equilibrium strategy does not change with the voting rule, and hence it occupies only a single column (right-hand column) in Table 2. The strategy is also identical to the optimal strategy for the singleDM ERSP with $n=40$; it rejects the first 11 alternatives before possibly accepting any.

Table 2 is supplemented by Figure 1, which exhibits a graphic representation of the equilibrium strategy for each possible voting rule at $\mu=0,1 / 3,2 / 3$, and 1 . Figure 1 shows that, with imperfect correlation $(\mu<1)$, as $q$ increases from one (minority rule) to two (majority rule) or three (unanimity rule) the threshold rank in equilibrium at any decision stage $(j)$ often decreases and sometimes remains the same, but never increases. That is, the committee member generally becomes less compromising when the number of votes required for stopping the search increases. For example, under the majority and unanimity rules, when $\mu=$ 0 the committee member will not accept the first and second inspected alternatives, and will accept an alternative among the third to fifth inspected alternatives only if it is the best so far.

By contrast, the change of threshold ranks from majority rule to unanimity rule is not of the same direction across different values of $j$. In general, it appears that with imperfect preference correlation there is a reversal as $j$ increases: when $j$ is low, the threshold ranks under the unanimity rule are lower than or equal to (but never higher than) those under the majority rule; when $j$ is high, the opposite becomes true. 
Table 2 Equilibrium Threshold Ranks for Three-Person Committees with $\mu=0$ and $\mu=1(n=40)$

\begin{tabular}{|c|c|c|c|c|}
\hline \multirow[b]{2}{*}{$J$} & \multicolumn{3}{|c|}{ Uncorrelated preferences $(\mu=0)$} & \multirow{2}{*}{$\begin{array}{l}\text { Perfectly correlated } \\
\text { preferences }(\mu=1) \\
(\text { same as single-DM) }\end{array}$} \\
\hline & Minority rule $(q=1)$ & Majority rule $(q=2)$ & Unanimity rule $(q=3)$ & \\
\hline 1 & 0 & 0 & 0 & 0 \\
\hline 2 & 1 & 0 & 0 & 0 \\
\hline 3 & 1 & 1 & 1 & 0 \\
\hline 4 & 2 & 1 & 1 & 0 \\
\hline 5 & 2 & 1 & 1 & 0 \\
\hline 6 & 3 & 2 & 1 & 0 \\
\hline 7 & 3 & 2 & 2 & 0 \\
\hline 8 & 4 & 2 & 2 & 0 \\
\hline 9 & 4 & 3 & 2 & 0 \\
\hline 10 & 5 & 3 & 3 & 0 \\
\hline 11 & 5 & 3 & 3 & 0 \\
\hline 12 & 5 & 3 & 3 & 1 \\
\hline 13 & 6 & 4 & 3 & 1 \\
\hline 14 & 6 & 4 & 4 & 1 \\
\hline 15 & 7 & 4 & 4 & 1 \\
\hline 16 & 7 & 5 & 4 & 1 \\
\hline 17 & 8 & 5 & 5 & 1 \\
\hline 18 & 8 & 5 & 5 & 1 \\
\hline 19 & 9 & 6 & 6 & 1 \\
\hline 20 & 9 & 6 & 6 & 2 \\
\hline 21 & 10 & 6 & 6 & 2 \\
\hline 22 & 10 & 7 & 7 & 2 \\
\hline 23 & 10 & 7 & 7 & 2 \\
\hline 24 & 11 & 7 & 8 & 2 \\
\hline 25 & 11 & 8 & 8 & 2 \\
\hline 26 & 12 & 8 & 8 & 3 \\
\hline 27 & 12 & 8 & 9 & 3 \\
\hline 28 & 13 & 9 & 9 & 3 \\
\hline 29 & 13 & 9 & 10 & 4 \\
\hline 30 & 14 & 10 & 11 & 4 \\
\hline 31 & 14 & 10 & 11 & 5 \\
\hline 32 & 15 & 11 & 12 & 5 \\
\hline 33 & 15 & 11 & 13 & 6 \\
\hline 34 & 16 & 12 & 13 & 7 \\
\hline 35 & 16 & 13 & 14 & 8 \\
\hline 36 & 16 & 14 & 15 & 9 \\
\hline 37 & 17 & 15 & 16 & 11 \\
\hline 38 & 18 & 17 & 18 & 14 \\
\hline 39 & 20 & 20 & 20 & 20 \\
\hline
\end{tabular}


Figure 1. Graphical Representations of the Equilibrium Threshold Ranks for Three-Person Committees at Different Levels of Preference Correlation $\mu(n=40)$.
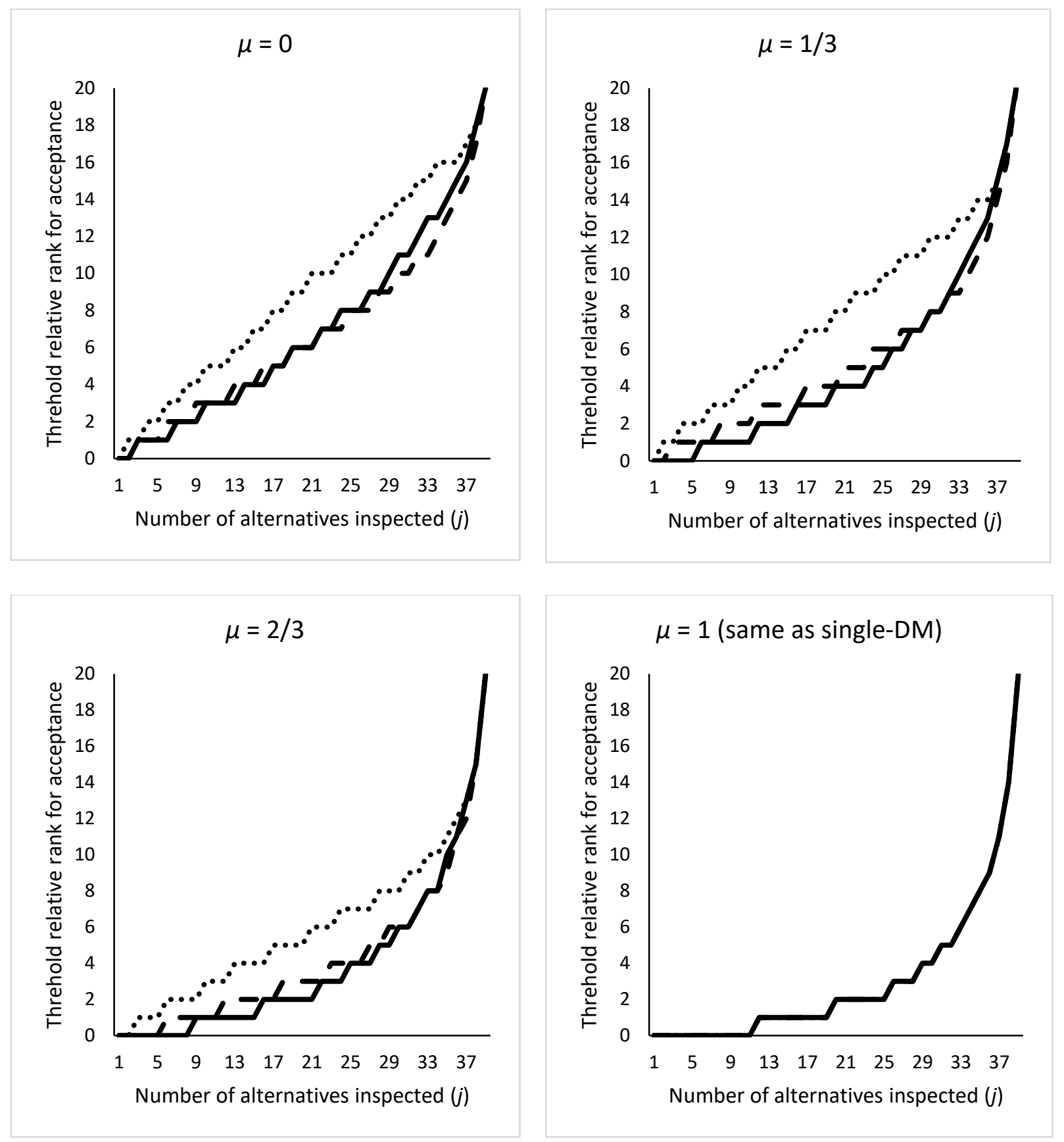

Note. The dotted line represents the minority rule, dashed line represents the majority rule, and solid line the unanimity rule. Note that when $\mu=1$ (perfectly correlated preferences), all the three lines coincide. 
Table 3 Equilibrium and Observed MAR and MSL Scores in the Three Experiments

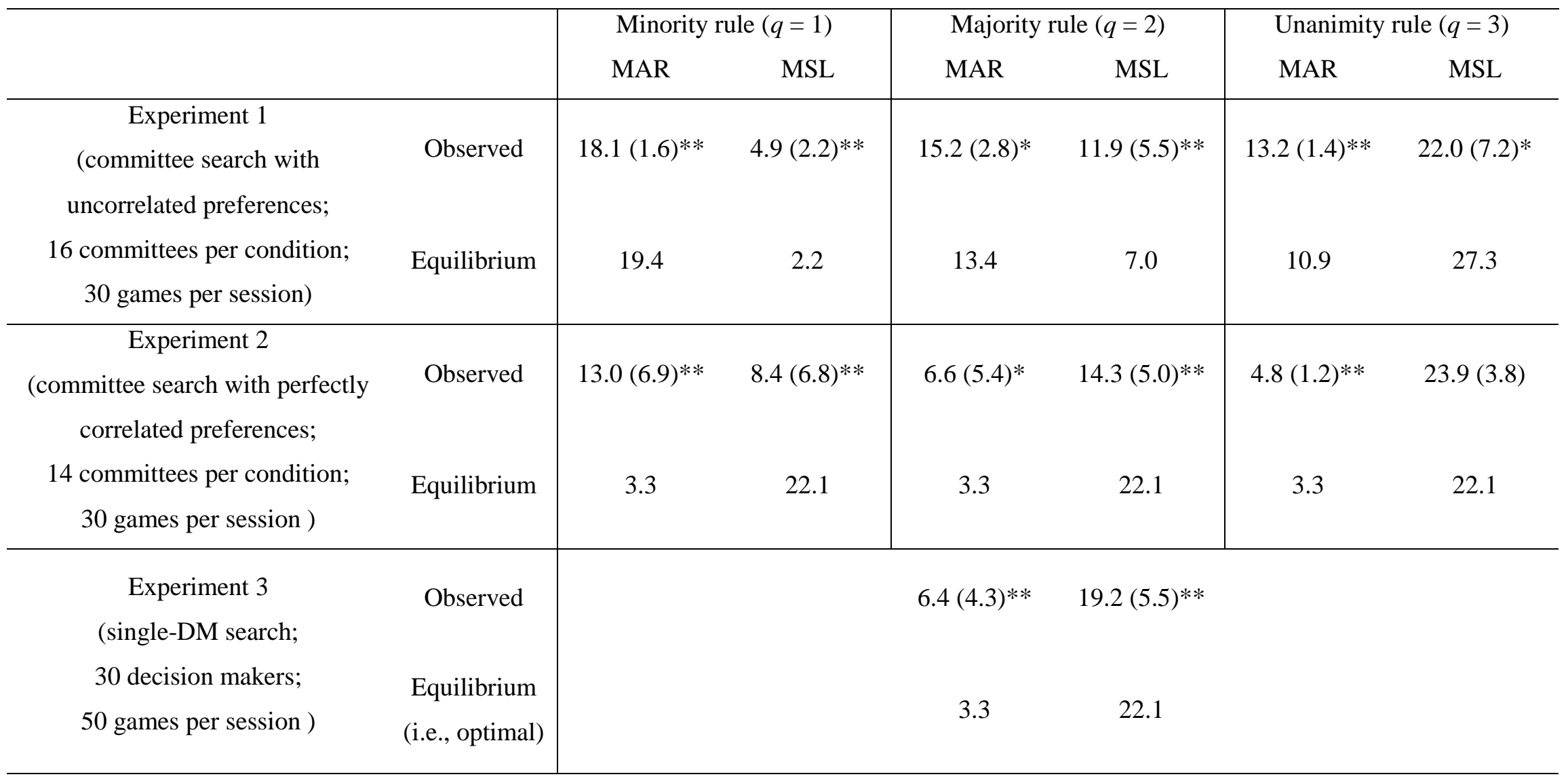

Note. SDs (with committee as the unit of analysis in Experiment 1 and 2 and individual subject as the unit of analysis in Experiment 3 ) are in parentheses. An observed mean with one or more asterisks indicates that the mean is significantly different from the relevant equilibrium prediction according to a $t$-test $(* p<0.05 ; * * p<0.01)$. 
For example, when $\mu=0$, this reversal happens from $j=24$ onwards. The intuition is that as the search approaches its natural termination, where all possibilities would have been exhausted, the committee members become more compromising under the unanimity than the majority rule - if they continue to be relatively intransigent and veto each other they might end up with an even less desirable alternative.

We next examine the expected values of two measures of search performance when $m=3$, with a focus on cases represented in our experiments. Table 3 lists the numerically computed equilibrium mean search length (MSL) of the committee and mean absolute rank (MAR) obtained by each committee member under every voting rule when the preferences are uncorrelated $(\mu=0)$ and perfectly correlated $(\mu=1)$, respectively. As $q$ increases, the search becomes longer on average, as committee members more often veto each other's decisions. At the same time, every committee member gains in achieving lower MAR. That is, as $q$ increases, the gain in the focal member's voting power over-compensates the concurrent gain in the other members' voting power, resulting in a net gain in benefit to this member.

When the committee members have perfectly correlated preferences, the case is theoretically equivalent to single-DM ERSP. There is no conflict arising from misaligned preferences; concurrently, members compromise less in the sense of accepting less desirable alternatives early on, resulting in longer MSL and lower MAR than when $\mu=0$. If all the committee members follow the equilibrium strategy, then they will either unanimously accept or unanimously reject any alternative, since the equilibrium strategy and the relative ranks are identical among all the members.

\section{Experiment 1: Committee Search with Uncorrelated Preferences}

In this and the next two sections, we report three experiments by which we seek behavioral evidence on how well DMs can approximate equilibrium strategies in our search decision context, and whether there are any systematic deviations equilibrium that vary with the voting rule. As explained before, we focus on a minimal committee size that still offers the possibilities of minority, majority, and unanimity voting rules. Experiments 1 and 2 involve three-person committees under all three possible voting rules; each experiment captures one end of the range of preference correlation, namely, $\mu=0$ (uncorrelated preferences) in 
Experiment 1, and $\mu=1$ (perfectly correlated preferences) in Experiment 2. Experiment 3 involves a similar search task but with only a single DM to provide comparison to the results in Experiment 1 and 2.

\subsection{Subjects}

One hundred and forty-four subjects with approximately equal proportions of males and females participated in the experiment. The subjects were mostly undergraduate business majors between 18 and 25 years of age at a southwestern university. This sample size was obtained based on a target of 16 committees (48 subjects) per voting rule condition, which was achieved. Subjects' earnings were contingent on their performance, and were on average \$21 including a \$5 show-up fee. Each session lasted approximately 90 minutes on average.

\subsection{Design}

The subjects were randomly assigned to three-person committees with fixed membership throughout the experiment. Implementing a between-subjects design, each committee participated in one of three experimental conditions defined by the voting rule, namely, minority rule, majority rule, and unanimity rule conditions. A research protocol indicating how the subjects would be treated and reimbursed was approved by the second author's Institutional Review Board (IRB).

\subsection{Procedure}

Upon entering the computer laboratory, the subjects were randomly assigned to workstations and provided with (i) an informed consent form that explained their rights, (ii) scratch paper to record any outcomes that they deemed important, and (iii) a copy of the instructions (see Online Appendix A.2 for a sample). The subjects were instructed that they would take part in a computer-based decision experiment as members of three-person committees. They participated in 30 games (trials, decision stages) of the sequential search game described below. The games were identical up to a different permutation of the $n$ ranks in each game.

A potential challenge of the experiment was to convey the idea of uncorrelated rankings to the subjects. Since terms like "uncorrelated" might be deemed too technical to laypersons, we avoided them in the instructions and stated to the subjects that "each member of your 3-person committee will have different relative and absolute ranks for the alternatives presented." To drive home the point, at the beginning of the 
instructions we compared the experimental task to the business purchase decision making of a managerial committee (in a context that subjects should be able to easily connect with), with which committee members might have different opinions of each product considered. Afterwards, in the main part of the instructions, we used more abstract wordings to avoid anchoring the subjects' decisions on any given context.

At every decision point during each search game, the subjects were not informed of their absolute ranks of the current alternative, but only of the relative rank of the current alternative with respect to previous alternatives. Moreover, the subjects were informed only of their own relative ranks of the alternatives, not of the relative ranks of the same alternatives for the other two committee members. They were further informed that their payoffs would depend on their absolute ranks of the alternative that was accepted by the committee.

After each alternative was presented and its relative rank disclosed, each committee member could either accept or reject it and submit the decision to the voting rule. The computer program then tracked the number of accept decisions in the committee. For committees participating under the minority rule condition, a single accept decision was sufficient to stop the search. For committees under the majority or unanimity rule conditions, at least two or exactly three accept decisions, respectively, were necessary to stop the search. If the committee rejected the first 39 alternatives in the sequence, then the $40^{\text {th }}$ alternative was accepted by default. At the end of each game, the absolute ranks were revealed, each subject was informed of the number of the alternative that was accepted, its absolute rank to him/her, and his/her own payoff for the game computed by the formula (30 - absolute rank) points, the experimental currency.

To minimize wealth effects, every subject's payment was determined by his/her earnings in five games that were randomly chosen at the end of the session (same games for all subjects in the same session), with the conversion rate $\$ 0.20=1$ point. The computer software privately displayed the subject's earnings for each of these five games. Then, the subjects were paid in cash and dismissed.

\subsection{Results and Analysis}

Non-significant learning effects. We first note the absence of significant learning effects during the experimental session. To demonstrate this, we divided the data into three blocks of 10 games each. We then 
conducted MANOVA in a 3 (condition) $\times 3$ (block) mixed design, where condition is the between- subjects factor and block is the within-subjects factor, with absolute rank of the accepted alternative and search length as the two dependent variables. The analysis did not reveal any significant effect due to block $(F(2,44)=0.65$, Wilks' $\lambda=0.97, p=0.53)$. We conclude that the major outcome measures, in terms of the two dependent variables, did not change significantly over the course of the experiment. It seems that the subjects' strategies had by and large settled down during the early rounds. Therefore, in the subsequent analysis we do not distinguish between different parts of the experimental session.

Committee-level analysis. Row 2 of Table 3 lists the major aggregate measures, namely, the MAR and MSL scores, with the committee as the statistical unit of analysis. The corresponding expected values under the equilibrium strategy are also listed, and statistically significant deviations of observed means from the equilibrium predictions are indicated by asterisks. For further reference, Online Appendix A.5 displays graphically more fine-grained performance data; Online Appendix A.6 reports average counts of realized searches that were incidents of over-search or under-search relative to what would have happened had subjects followed equilibrium strategies. These appendices jointly with Table 3 suggest the following:

(1) Deviations from benchmark predictions: contrary to previous literature in which under-search relative to the equilibrium strategy was prevalent (e.g. Bearden and Rapoport 2005), deviations from equilibrium predictions were characterized by over-search under the minority and majority rules, but undersearch under the unanimity rule. The observed absolute ranks were inferior to the equilibrium predictions except under the minority rule, when the MAR was significantly better (i.e., lower) than under equilibrium.

(2) Performance comparisons under different voting rules: the observed MAR was minimized (i.e., optimized) under the unanimity rule. Pairwise $t$-test comparisons between the MARs under the unanimity rule and each of the other two conditions support our claim at $p<0.01$. That is, the committees in this experiment made the best decisions (in terms of committee welfare) under the unanimity rule. This is consistent with theory, though also possibly driven by behavioral factors.

Note that the subjects could not have been using simple randomization decision rules - had they been using such rules, the average absolute rank would have been close to $20.5(=(1+2+\ldots 40) / 40)$ regardless of 
Table 4 Individual Decision Level Deviation Rate in the Three Experiments

\begin{tabular}{|c|c|c|c|c|c|c|c|c|c|}
\hline & \multicolumn{3}{|c|}{ Minority rule $(q=1)$} & \multicolumn{3}{|c|}{ Majority rule $(q=2)$} & \multicolumn{3}{|c|}{ Unanimity rule $(q=3)$} \\
\hline & \multicolumn{9}{|c|}{ Proportion of individual decisions with (relative to (a) equilibrium and (b) empirical best response strategy) ... } \\
\hline & $\begin{array}{c}\text { Type I } \\
\text { deviation }\end{array}$ & $\begin{array}{c}\text { No } \\
\text { deviation }\end{array}$ & $\begin{array}{c}\text { Type II } \\
\text { deviation }\end{array}$ & $\begin{array}{c}\text { Type I } \\
\text { deviation }\end{array}$ & No deviation & $\begin{array}{c}\text { Type II } \\
\text { deviation }\end{array}$ & $\begin{array}{c}\text { Type I } \\
\text { deviation }\end{array}$ & No deviation & $\begin{array}{c}\text { Type II } \\
\text { deviation }\end{array}$ \\
\hline $\begin{array}{ll} & \text { (a) } \\
\text { Expt. } 1 & \text { (b) }\end{array}$ & $\begin{array}{l}0.02(0.03) \\
0.02(0.04)\end{array}$ & $\begin{array}{l}0.70(0.05) \\
0.71(0.04)\end{array}$ & $\begin{array}{l}0.28(0.06) \\
0.27(0.05)\end{array}$ & $\begin{array}{l}0.09(0.08) \\
0.07(0.07)\end{array}$ & $\begin{array}{l}0.80(0.08) \\
0.75(0.08)\end{array}$ & $\begin{array}{l}0.11(0.05) \\
0.18(0.05)\end{array}$ & $\begin{array}{l}0.16(0.07) \\
0.14(0.07)\end{array}$ & $\begin{array}{l}0.78(0.07) \\
0.78(0.07)\end{array}$ & $\begin{array}{l}0.06(0.02) \\
0.08(0.03)\end{array}$ \\
\hline Expt. $2^{\text {(a) }}$ & $\begin{array}{l}0.11(0.14) \\
0.09(0.14)\end{array}$ & $\begin{array}{l}0.89(0.13) \\
0.84(0.11)\end{array}$ & $\begin{array}{l}0.01(0.01) \\
0.07(0.04)\end{array}$ & $\begin{array}{l}0.12(0.14) \\
0.10(0.14)\end{array}$ & $\begin{array}{l}0.88(0.14) \\
0.88(0.14)\end{array}$ & $\begin{array}{l}0.01(0.01) \\
0.02(0.01)\end{array}$ & $\begin{array}{l}0.16(0.15) \\
0.14(0.14)\end{array}$ & $\begin{array}{l}0.83(0.15) \\
0.82(0.14)\end{array}$ & $\begin{array}{l}0.01(0.01) \\
0.03(0.01)\end{array}$ \\
\hline Expt. 3 (a) & & & & $0.03(0.04)$ & $0.96(0.03)$ & $0.01(0.01)$ & & & \\
\hline
\end{tabular}

Note. A Type I deviation (“false positive") occurs when a decision maker accepts an alternative while the relevant benchmark strategy prescribes rejecting it. A Type II deviation ("false negative") occurs when a decision maker rejects an alternative while the relevant benchmark strategy prescribes accepting it. The means and the SDs (in parentheses) are calculated with a committee as the unit of analysis in Experiment 1 and 2 and individual subject as the unit of analysis in Experiment 3. The sum of the three means for the same benchmark in any condition is (up to rounding errors) equal to one, as expected. 
the number of searches. Table 3 shows that this was not the case ( $t$-tests confirm that each MAR in row 2 of Table 3 is significantly smaller than 20.5 at $p<0.01$ ). There is also no evidence of committee members colluding to maximize the total expected payoff of the committee. For the majority and unanimity rule conditions, had the members been colluding, their mean expected payoff could not have been worse than the equilibrium average. In fact, their payoffs were significantly worse (i.e., the MARs were higher) than in equilibrium in those conditions. For the minority rule condition, we reason that a collusive outcome must not be worse than the scenario in which every committee member played the ERSP optimal strategy (the right-hand column of Table 2). Numerical computation shows that the predicted MAR and MSL scores in this scenario are 15.1 and 15.8, respectively. The observed MAR and MSL scores are significantly different from these predictions according to $t$-tests ( $p<0.01$ in both tests). In particular, the observed MAR score is significantly worse than the predicted value. We thus conclude that there is also no evidence of collusion in the minority rule condition.

Individual-level analysis. We next carried out a more fine-grained analysis of committee members' individual decisions throughout the searches. For every observed decision, we compared it to the one prescribed by equilibrium strategy; wherever a deviation from equilibrium play was detected, we distinguished between two types of deviations: a Type I ("false positive") deviation occurs when a DM accepts an alternative while equilibrium strategy prescribes rejecting it; a Type II ("false negative") deviation occurs when a DM rejects an alternative while the equilibrium strategy prescribes accepting it. In the analysis, we first computed for every committee the proportion of these deviations among all decisions made by its members, and then calculated the mean of these proportions across committees in each condition. Row 4 in Table 4 summarizes our results.

Best Response Analysis. In addition, we tested the individual decisions against an estimated empirical best response strategy with respect to the population. Specifically, for each voting rule condition, the empirical best response strategy involves prescribing a threshold relative rank $r_{j}$ for the $j$-th alternative $(j=1,2,3 \ldots 39)$, such that a DM $i$ should reject the $j$-th alternative if and only if the relative rank of the alternative, $R_{i, j}$, is higher (i.e., worse) than $r_{j}$. We determined the value of $r_{j}$ as follows: 
(1) For each experimental committee, we computed the mean absolute rank obtained by the committee members in all the searches (if any) that stopped after inspecting more than $j$ alternatives;

(2) We then computed the mean of these average absolute ranks among all the committees in the same voting rule condition. We used this mean as a proxy for the empirical "continuation expected payoff" in the experimental population when one of its committees rejected the $j$-th alternative. The continuation expected payoff may be perceived as the empirical counterpart of $\bar{U}_{i, j}$ in the equilibrium analysis;

(3) If committee member $i$ best responded to the experimental population, then he/she should vote to accept/reject the $j$-th alternative if its expected absolute rank is superior/inferior to the continuation expected payoff. As noted earlier, if the $j$-th alternative has relative rank $R_{i, j}$, its expected absolute rank is equal to $(n+1) R_{i, j} /(j+1)$. This relationship then allows us to obtain the threshold relative ranks $r_{j} \mathrm{~s}$ for the empirical best response strategy.

The empirical best response strategy thus computed is summarized in Online Appendix A.7 as a series of thresholds relative ranks for each voting rule. The differences with the corresponding equilibrium threshold relative ranks are also listed; Appendix A.7 shows that they are typically only one or two ranks apart. This suggests that testing subject decisions against equilibrium or empirical best response strategies should yield similar results. This is borne out in Table 4, as the deviations in rows (a) (relative to equilibrium) and (b) (relative to empirical best response) are very similar in each voting rule condition.

Two major observations are highlighted by this analysis. First, individual decisions were consistent with either equilibrium or empirical best response benchmark for around 70\%-80\% of the times; note that since this analysis involved all the decisions that occurred during the search, individual-level decisions could appear to be much more consistent with strategic benchmarks than the MSL figures in Table 3 might imply. This is because a single deviation after a string of decisions following equilibrium could disproportionately register as one incident of over- or under-search. Secondly, there is evidence of an increase in the "no deviation" measures as the voting rule changed from minority to majority (pairwise $t$ tests for the equilibrium and empirical best response measures yield $p<0.01$ and $p<0.1$, respectively). 
The measure then remained approximately the same as the voting rule changed from majority to unanimity ( $t$-tests for both measures yield $p>0.15$ ). Concurrently, Type II deviation rates decreased while Type I deviation rates increased, which are consistent with the change from over-search to under-search.

Discussion. Why did these committee- and individual-level differences across conditions occur? A possible answer is that, as discussed earlier, the unanimity rule condition is the most strategically complex of the three because the subjects could veto each other to prolong search and invite more conflicts. In fact, the observed proportional frequency of a member's decision being in conflict with the committee decision (i.e., either the member accepted an alternative that was rejected by the committee, or vice versa) was $14.9 \%$ and $16.4 \%$ under the minority and majority rule conditions, respectively, but rose sharply to $31.5 \%$ under the unanimity rule condition. Using the committee as the statistical unit of analysis, statistical analysis provides converging evidence that this proportion was significantly higher under the unanimity rule than under either of the other two rules ( $p<0.01$ by $t$-tests for both comparisons).

Therefore, it might be expected that the subjects in the unanimity rule condition had perceived more strongly the strategic complexity of the game and had been much more sensitized to its strategic aspects than in the two other conditions. If those subjects had been over-sensitized, then they might be too ready to accept a mediocre alternative early (relative to the equilibrium strategy) in order to pre-empt future conflicts, leading to under-search. Meanwhile, being not sufficiently sensitized to the strategic aspects of the game, subjects in the other two conditions might be expected to attempt too little to accept a compromising alternative to pre-empt future conflicts.

Sensitization to strategic complexity also appeared to drive Mak et al. (2014)'s findings that information flow between members of two-person committees could reduce over-search. In more general terms, previous research shows that people could adopt different decision rules under different situations the so-called "adaptive toolbox" perspective (see, e.g., Payne, Bettman, and Johnson 1988, Gigerenzer and Todd 1999, and the overview in Bröder and Newell 2008). A premise of that literature is that individuals make tradeoffs between cognitive effort and the demand of the task environment. Our results may thus be interpreted as subjects trading off cognitive effort and the demand of the task in terms of the perceived 
strategic complexity, so that they invested more cognitive effort when the perceived strategic complexity increased to the effect of a reduction in deviations from equilibrium. Relatedly, Agranov et al. (2012) show that individuals' strategic sophistication could be sensitive to the environment; here we present evidence supporting this finding in committee decision making in a sequential search task.

\section{Experiment 2: Committee Search with Perfectly Correlated Preferences}

Experiment 2 was designed to investigate sequential search behavior in three-person committees under three different voting rules when committee members' rankings were perfectly correlated $(\mu=1)$. The decision task was identical to the single-DM ERSP except that a committee, rather than individual, made decisions on alternatives on which their preferences were completely aligned. The experiment served two major purposes. First, it established behavioral findings for a context that was opposite from that in Experiment 1 in terms of preference correlations. Secondly, if strategic complexity in Experiment 1 due to misaligned preferences drove our findings, then we would expect to observe dissimilar results in Experiment 2. Specifically, because preferences among committee members in Experiment 2 were completely aligned, there would not be any conflict arising from misaligned preferences, and any strategic complexity factors resulting from such conflict - and subsequent differences across voting rules as discussed previously - would not be present in Experiment 2.

\subsection{Subjects}

One hundred and twenty-six subjects with approximately equal proportions of males and females participated in the experiment. The subjects were students at a southwestern university that was different from the one in Experiment 1, but otherwise had similar demographic characteristics. The sample size was obtained to reach a target of 14 committees (42 subjects) per voting rule condition, which was achieved. Subjects' earnings were on average \$27 including a \$5 show-up fee. In addition, the subjects earned an average $\$ 4$ from the post-experimental questionnaire. Each session lasted approximately 90 minutes.

\subsection{Design}

The design was the same as that in Experiment 1. IRB approval was obtained.

\subsection{Procedure}


The procedure was identical to Experiment 1 except that all the three committee members observed the same relative rank for the same alternative. The instructions, a sample of which appears in Online Appendix A.3, are the same as those in Experiment 1, except that there is less elaboration on the motivating business example at the beginning. This is because perfectly correlated preferences are easier to convey than uncorrelated preferences. On the other hand, an additional feature was a post-experimental questionnaire (see Online Appendix A.3), in which the subjects were quizzed on some aspects of the decision task. We used the questionnaire to confirm understanding of the task. To increase involvement in the task, we incentivized the subjects by paying out $\$ 1$ for every correct answer.

\subsection{Results and Analysis}

Detailed analysis of the questionnaire data (see Online Appendix A.8) shows that the subjects' understanding of the decision task was uniformly high across conditions; such level of understanding was achieved early in the sessions. This finding is consistent with the fact that we have not observed significant learning effects in terms of other performance variables. Following the similar analysis for Experiment 1, a 3 (condition) $\times 3$ (block) mixed-design MANOVA on absolute rank of accepted alternative and search length did not yield any significant effect due to block $(\mathrm{F}(2,38)=2.24$, Wilks' $\lambda=0.89, p=0.12)$. We conclude that the two major outcome measures MAR and MSL did not change significantly over the course of the experiment.

Committee-level analysis. Our committee-level analysis suggests the following:

(a) Deviations from benchmark predictions: Row 4 of Table 3 shows that there was overall undersearch relative to the equilibrium in the minority and majority rule conditions, but search length was approximately equal to the equilibrium expected value in the unanimity rule condition. Search payoff (in terms of MAR) was always significantly inferior to the equilibrium. These observations are consistent with the plots in Online Appendix A.5, and the committee-level realized search deviation data in Online Appendix A.6. Noting that collusive behavior would have been equivalent to equilibrium behavior in this experiment, there is no evidence of collusion. 
(b) Performance comparisons under different voting rules: As the number of votes required to accept an alternative increased across conditions, there seems to be a decrease in deviations and increase in search performances approaching equilibrium - despite the equilibrium predictions being identical across conditions. Pairwise $t$-tests show that the MSL scores increased significantly at $p<0.05$ from minority to majority as well as from majority to unanimity rules, so that in the last condition the MSL reached the equilibrium prediction. Meanwhile, the MAR scores improved significantly from the minority to the majority rule at $p<0.05$ but were not significantly different between the majority and unanimity rules $(p=$ 0.22). This means that the committees under the unanimity rule searched more like they were following equilibrium strategies, but without gaining substantial improvement in their outcome compared with those under the majority rule. Overall, in line with our general theoretical conclusions, as well as Experiment 1, the optimal voting rules for the subjects in Experiment 2 required at least a majority to terminate the search.

Individual-level analysis. We performed deviation rate analysis with respect to the equilibrium and empirical best response strategy, as with Experiment 1; the results are summarized in Table 4. In contrast with Experiment 1, there were no notable changes in all the deviation measures as the voting rule changed ( $p>0.2$ in all relevant pairwise comparisons using $t$-tests). The changes with voting rule (in terms of MSL) seemed to have been caused by the voting rule rather than inherent differences in the strategic sophistication of individual decisions. Specifically, even if the subjects followed equilibrium decision at the same rate under different voting rules, if their deviations were mostly Type I ("false positives") - which is indeed the case from Table 4 - then the unanimity rule would be more effective in reducing the likelihood of individual deviations becoming committee-level deviations in comparison with the other rules.

To sum up, without preference misalignment and the implied strategic complexity, decisions in Experiment 2 exhibited different biases from those in Experiment 1: there was no over-search, but only under-search and approximately optimal search. These results are understandable with the predominance of Type I deviations among individual decisions. These deviations could be more effectively reduced at the committee level as the number of subjects' votes needed to stop the search increased. Compared with the 
minority voting rule, the majority and unanimity rules seemed to function better as a check against the main tendency of the individuals to under-search and achieved significantly superior committee welfare.

\section{Experiment 3: Single-Decision Maker Search}

Experiment 3 was designed to provide further benchmarking for the observations in the previous experiments by investigating single-DM search behavior with the same ERSP setup. Experiment 3 did not have the strategic complexity driven by misaligned preferences; and its DMs were not subject to the need for any amount of consensus from other DMs to adopt a decision during the search process. We thus expect that behavior in Experiment 3 would exhibit an under-search pattern as in previous studies (see Section 2).

\subsection{Subjects}

Thirty subjects with approximately equal proportions of males and females participated in the experiment. The subjects were students at the same southwestern university as in Experiment 2. The sample size was obtained based on a target of 30 subjects, which was achieved. Subjects' earnings from the experiment were contingent on their performance, and were on average \$24 including a \$5 show-up fee. Each session lasted approximately 40 minutes.

\subsection{Design}

The design was a one-condition single-DM version of Experiment 2. IRB approval was obtained.

\subsection{Procedure}

With the exception of voting, the procedure was identical to Experiment 2. The instructions, a sample of which appears in Online Appendix A.4, are similar to those in Experiment 2 but in an individual context without any description related to committee search or voting rule. Because the task was considerably simpler in comparison to the committee search tasks in the other two experiments, we increased the number of games per session from 30 to 50 to ensure that the overall amount of decision experience of the subjects was approximately the same across all three experiments. Because of this increase in the number of decisions, we made the payoff calculation more direct: the payoff from a game was not computed in terms of an experimental currency but, rather, in dollars according to the formula ( $\$ 9$ - absolute rank).

\subsection{Results and Analysis}


Following the learning analysis for the previous two experiments, a 3 (condition) $\times 5$ (block) mixed-design MANOVA on absolute rank of accepted alternative and search length did not yield any significant effect due to block $(F(4,26)=0.83$, Wilks' $\lambda=0.89, p>0.5)$. We conclude that the major outcome measures, in terms of the two dependent variables MAR and MLS, did not change significantly over the course of Experiment 3. As such, we proceed to report analyses using all the search data in the experiment.

Similarly to Experiment 2, in Experiment 3 the subjects under-searched (note that the equilibrium predictions are identical to those for Experiment 2). This is attested by statistical analysis of the aggregates in Row 6 of Table 3. Search length (in terms of MSL) was significantly lower than equilibrium, while search payoff (in terms of MAR) was significantly inferior to equilibrium. These observations are consistent with the plots in Online Appendix A.5, which exhibit fine-grained committee-level search performance data in Experiment 3, and the deviation count data for realized searches in Online Appendix A.6.

Table 4 shows that individual decisions in Experiment 3 were highly consistent with equilibrium, in fact at a rate of $96 \%$. But note that, since the searches in this experiment were relatively long with an observed MSL of 19.2 and predicted MSL of 22.1, an under-search observation could result from a sequence of, say, 19 decisions following equilibrium, plus one single deviation that terminated the search too soon. Table 4 also shows that deviations from equilibrium in Experiment 3 leaned towards Type I, which is consistent with the under-search observations at the committee level.

\section{Comparisons across Experiments}

This section reports results arising from comparisons across the three experiments. These results offer insights into, e.g., whether committees performed better or worse than single DMs in our search task.

Table 4 suggests that, controlling for the voting rule, committee members' individual decisions were less strategic when preferences were uncorrelated than when they were perfectly correlated. To examine this observation, we conducted pairwise $t$-tests of the equilibrium "no deviation" measure across Experiments 1 and 2 for each voting rule, and then similar $t$-tests of the empirical best response "no deviation" measure. We find that both measures increased significantly from Experiment 1 to Experiment 2 under the minority rule ( $p<0.01$ in both tests), and this remained largely the case under the majority rule 
( $p \approx 0.06$ for the equilibrium measure, and $p<0.01$ for the empirical best response measure), but the differences were non-significant under the unanimity rule ( $p>0.2$ for both measures). These results reflect the fact that committee sequential search with uncorrelated preferences is a strategically more complex scenario than sequential search with perfectly correlated preferences, so that subjects exhibit less strategic sophistication in the former - an occurrence that was noticeably mitigated under the unanimity rule.

Table 4 shows that the "no deviation" measure in Experiment 3, with a mean of 0.96, is significantly higher than the corresponding mean in any voting rule condition in Experiments 1 and $2(p<0.01$ in all relevant $t$-test comparisons). Whereas the differences with Experiment 1 may be intuited from the difference in strategic complexity, the contrast with Experiment 2 is more intriguing. It is possible that when making decisions in a committee with highly aligned preferences, members develop a dependency on each other's strategic sophistication and, therefore, are more relaxed in their own strategic considerations. This finding echoes previous studies on how strategic sophistication could change with the environment (Agranov et al. 2012); the psychological factors behind this finding merit further investigation.

Consistently, the MSL score of Experiment 3 was longer than in the minority and majority rule conditions of Experiment 2, but shorter than in the unanimity rule condition of that Experiment (all pairwise $t$-tests yielding $p<0.01)$. Meanwhile, the MAR score of Experiment 3 was lower than (i.e., superior to) the MAR score in the minority rule condition of Experiment $2(t(42)=-3.90, p<0.01)$ but not significantly different from the MAR of the other two voting rule conditions ( $p>0.05$ in both comparisons). To sum up, committees' collective decisions were more strategic than individual decisions only under the unanimity rule, though still not significantly better in terms of the decision makers' welfare.

\section{Overall Methodological Issues}

Our study explores a new, distinctive, and hitherto unexplored set of research questions. This leads to methodological questions such as false positives in hypothesis testing (Maniadis, Tufano, and List 2014). Meanwhile, the large number of significant deviations from equilibrium also call for considerations of familywise errors in multiple hypothesis testing (List, Shaikh, and Xu 2016). In Online Appendix A.9, we report analyses suggesting that our major findings are robust upon consideration of both types of issues. 


\section{Discussion and Managerial Implications}

We orientate our concluding discussion and the managerial implications of our findings around our three major research objectives (see Section 1):

(1) What kind of decision improvement effort will be desirable to committee decision making?

Adopting the equilibrium as a prescriptive benchmark (see the Introduction section), we find that when preferences were uncorrelated committee members over-searched under the minority and majority voting rule but tended to under-search under the unanimity voting rule. When the preferences were perfectly correlated or when there was only a single DM, the subjects under-searched or approximated equilibrium play. These suggest that decision improvement efforts should: (i) promote compromising decisions (i.e., to curb over-search) when the committee members have weakly aligned or uncorrelated preferences and the voting rule does not require a high threshold number of votes to stop the search; (ii) promote more rejections (i.e., to avoid under-search) otherwise. Our experimental findings also suggest that improvement in strategic sophistication is more important when the committee members' preferences are more diverse.

(2) Does a committee with strongly aligned or perfectly correlated preferences necessarily make better decisions than an individual? Conventional wisdom might assume that a committee with strongly aligned or perfectly correlated preferences should be able to make better (i.e., more optimal) collective decisions for the committee's (and thus every member's) welfare compared with an individual in a similar task making decisions for his/her own welfare. Our results suggest that this assumption is contingent on how individual decisions are aggregated into the committee decision. In our experiments, at a collective level, committees with perfectly correlated preferences made decisions that were closer to benchmark predictions on optimal search behavior (i.e., "smarter") than individuals only under the unanimity rule and there was no significant welfare improvement over single DM search even in that case. That is, the committee's collective decisions might be "smarter" than the individual's in that case, but not significantly better. The results are driven by the fact that, at an individual level, committee members suffered from a deterioration in strategic sophistication compared with individual decision makers' even under perfectly 
correlated preference. Our findings caution against simplistic assumptions about the quality of committee decision making over individual decision making.

(3) What might be the best voting rule for aggregating individual decisions into the collective committee decision? This question is related to how to design the committee decision process to improve outcomes. We find that when preferences were uncorrelated, committees made the best decisions (in terms of committee welfare) under the unanimity voting rule - which is consistent with theoretical predictions. On the other hand, when preferences were perfectly correlated, in theory the voting rules should all have yielded the same level of welfare; but in Experiment 2, compared with the minority voting rules, the majority and unanimity rules seemed to be able to function better as a check against the main tendency of individual DMs to under-search, and achieved significantly superior committee welfare. As a rule, across Experiments 1 and 2, the unanimity rule always optimized committee welfare.

\subsection{Future Directions}

Our paradigm may be extended in several directions. For example, there is merit in studying situations where voting power is distributed unevenly among committee members in the form of differential weights of votes. In the extreme case, one member might be a "dictator" with predominant voting weight. It would be interesting to see how other committee members' preferences and welfare will or will not be considered by the "dictator"; also, if the other committee members could provide feedback to the "dictator" over the inspected alternative, how the "dictator" might incorporate such feedback into his/her own decision.

Another potential avenue is optimal voting rule. Our numerical calculations show that for two- or three-person committees the equilibrium total expected payoff (i.e., welfare) of the committee is optimized under the unanimity rule. This is consistent with our experimental findings. However, for larger committees (see Online Appendix A.10 for a set of examples), the optimal voting rule is generally a supermajority rule, in which the threshold number of votes to stop the search is larger than simple majority but falls short of unanimity. These results for larger committees apparently hold regardless of preference correlation; it would be important to test them experimentally; overall, future experimentation with larger committees could yield fruitful insights on how committee decision quality might differ according to the voting rule. 


\section{References}

Abdelaziz, F. B., Krichen, S. (2007). Optimal stopping problems by two or more decision makers: A survey. Computational Management Science 4: 89-111.

Agranov, M., Potamites, E., Schotter, A., Tergiman, C. (2012). Beliefs and endogenous cognitive levels: An experimental study. Games and Economic Behavior 75: 449-463.

Albrecht, J., Anderson, A., Vroman S. (2010). Search by committee. Journal of Economic Theory 145: 1386-1407.

Bearden, J. N., Murphy, R. O., Rapoport, A. (2005). A multi-attribute extension of the secretary problem: Theory and experiments. Journal of Mathematical Psychology 49: 410-425.

Bearden, J. N., Rapoport, A. (2005). Operations research in experimental psychology. In J. C. Smith (Ed.), Tutorials in operations research, 2005 (pp. 213-236). INFORMS.

Bearden, J. N., Rapoport, A., Murphy, R. O. (2006). Sequential observation and selection with rankdependent payoffs: An experimental test of alternative decision rules. Management Science 52: 1437-1449.

Black, D. (1958). Theory of Committees and Elections. Cambridge: Cambridge University Press.

Braunstein, Y. M., Schotter, A. (1982). Labor market search: An experimental study. Economic Inquiry 12: 133-144.

Bröder, A., Newell, B. R. (2008). Challenging some common beliefs: Empirical work within the adaptive toolbox metaphor. Judgment and Decision Making 3: 205-214.

Brown, M., Flinn, C. J., Schotter, A. (2011). Real-time search in the laboratory and the market. American Economic Review 101: 948-974.

Charness, G., Sutter, M. (2012). Groups make better self-interested decisions. Journal of Economic Perspectives 26: 157-176.

Chow, Y. S., Moriguti, S., Robbins, H., Samuels, M. (1964). Optimal solution based on relative ranks (the “secretary problem”). Israel Journal of Mathematics 2: 81-90. 
Cox, J., Oaxaca, R. L. (1989). Laboratory experiments with a finite-horizon job-search model. Journal of Risk and Uncertainty 2: 301-330.

Ferguson, T. S. (1989). Who solved the secretary problem? Statistical Science 4: 282-296.

Freeman, P. R. (1983). The secretary problem and its extensions: A review. International Statistics Review 51: 189-206.

Gigerenzer, G., Todd, P. M. (1999). Fast and frugal heuristics: the adaptive toolbox. In G. Gigerenzer, P., M. Todd and the ABC Research Group (Eds.), Simple Heuristics That Make Us Smart (pp. 3-34). New York: Oxford University Press.

Harrison, G. W., Morgan, P. (1990). Search intensity in experiments. Economic Journal 100: 478-486.

Hizen, Y., Kawata, K., Sasaki, M. (2013). An experimental test of a committee search model. European Economic Review 61: 59-76.

Inukai, K., Kawata, K., Sasaki, M. (2017). Committee search with ex-ante heterogeneous agents: Theory and experimental evidence. Institute of Labor Economics Discussion Paper Series, IZA DP No.10670.

Lindley, D. V. (1961). Dynamic programming and decision theory. Applied Statistics 10: 39-51.

Lippman, S. A., McCall, J. J. (1976). The economics of job search: A survey. Economic Inquiry, 14(2): 155-89, 347-68.

List, J. A., Shaikh, A. M., Xu, Y. (2016). Multiple hypothesis testing in experimental economics. NBER Working Paper Series, Working Paper 21875, http://www.nber.org/papers/w21875.

Mak, V., Rapoport, A., Seale, D. A. (2014). Sequential search by committees with rank-dependent payoffs: An experimental study. Organizational Behavior and Human Decision Processes 124: 256-267.

Maniadis, Z., Tufano, F., List, J. A. (2014). One swallow doesn’t make a summer: New evidence on anchoring effects. American Economic Review 104: 277-290.

Nurmi, H. (1987). Comparing Voting Systems. Dordrecht, Holland: D. Reidel.

Palley, A. B., Kremer, M. (2014). Sequential search and learning from rank feedback: Theory and experimental evidence. Management Science 60: 2525-2542. 
Payne, J. W., Bettman, J. R., Johnson, E. J. (1988). Adaptive strategy selection in decision making. Journal of Experimental Psychology: Learning, Memory, and Cognition 14: 534-552.

Rogerson, R., Shimer, R., Wright R. (2005) Search-theoretic models of the labor market: A survey. Journal of Economic Literature XLIII: 959-988.

Sakaguchi, M., Mazalov, V. V. (2004). A non-zero-sum no-information best-choice game. Mathematical Methods of Operations Research 60: 437-451.

Samuels, S. M. (1991). Secretary problems. In B. K. Ghosh and P. K. Sen (Eds.), Handbook of Sequential Analysis (pp. 381-405). New York: Marcel Dekker.

Schotter, A., Braunstein, Y. M. (1981). Economic search: An experimental study. Economic Inquiry 19: 125.

Schunk, D. (2009). Behavioral heterogeneity in dynamic search situations: Theory and experimental evidence. Journal of Economic Dynamics and Control 33: 1719-1738.

Schunk, D., Winter, J. (2009). The relationship between risk attitudes and heuristics in search tasks: A laboratory experiment. Journal of Economic Behavior and Organization 71: 347-360.

Seale, D. A., Rapoport, A. (1997). Sequential decision making with relative ranks: An experimental investigation of the "secretary problem". Organizational Behavior and Human Decision Processes 69: 221-236.

Seale, D. A., Rapoport, A. (2000). Optimal stopping behavior with relative ranks: The secretary problem with unknown population size. Journal of Behavioral Decision Making 13: 391-411.

Sonnemans, J. (1998). Strategies of search. Journal of Economic Behavior and Organization 35: 309-332.

Stein, W. E., Seale, D. A., Rapoport, A. (2003). Analysis of heuristic solutions to the best choice problem. European Journal of Operational Research 51: 140-152.

Sutter, M., Czermak, S., Feri, F. (2013). Strategic sophistication of individuals and teams. Experimental evidence. European Economic Review 64: 395-410.

Zwick, R., Rapoport, A., Lo, A. K. C., Muthukrishnan, A. V. (2003). Consumer search: not enough or too much? Marketing Science 22: 503-519. 


\section{Voting Rules in Sequential Search by Committees: Theory and Experiments}

\section{Online Appendix}

\section{A.1. Pairwise Correlation of Relative Ranks between Any Two Committee Members}

Consider two different committee members, $i$ and $i^{\prime}$, and their relative ranks $R_{i, j}$ and $R_{i^{\prime}, j}$ for the $j$-th alternative $(j>1)$ with respect to previous alternatives. As noted in the main text, the marginal distribution of either relative rank - the calculation of which involves aggregating over all possible $\bar{R}_{j} \mathrm{~s}-$ is a discrete uniform distribution over $\{1,2, \ldots j\}$. Thus, we may apply standard results for discrete uniform distributions to obtain:

$$
E\left(R_{i, j}\right)=E\left(R_{i^{\prime}, j}\right)=\frac{j+1}{2} \text { and } \operatorname{Var}\left(R_{i, j}\right)=\operatorname{Var}\left(R_{i, j}\right)=\frac{j^{2}-1}{12}
$$

Next, define:

$$
\lambda \equiv \frac{(1-\sqrt{\mu})(j-1)}{j}
$$

so that, conditioned on a realized value of $\bar{R}_{j}$, say $R$ :

$$
\begin{aligned}
& P\left(R_{i, j}=R \mid \bar{R}_{j}=R\right)=1-\lambda, \text { and } \\
& P\left(R_{i, j}=R^{\prime} \mid \bar{R}_{j}=R\right)=\lambda /(j-1) \text { for } R^{\prime} \in\{1,2, \ldots j\} \backslash\{R\},
\end{aligned}
$$

and similarly for $R_{i^{\prime}, j}$. Note that the expected value of $R_{i, j}$ conditioned on it being not $R$ is:

$$
\frac{(1+2+\ldots j)-R}{j-1}=\frac{1}{j-1}\left[\frac{j(j+1)}{2}-R\right]
$$

Hence: 


$$
\begin{aligned}
E\left(R_{i, j} R_{i^{\prime}, j} \mid \bar{R}_{j}=\right. & R)=(1-\lambda)^{2} R^{2}+\frac{2 \lambda(1-\lambda)}{j-1} R \cdot\left[\frac{j(j+1)}{2}-R\right]+\frac{\lambda^{2}}{(j-1)^{2}}\left[\frac{j(j+1)}{2}-R\right]^{2} \\
& =\left[\frac{j-1-\lambda j}{j-1} \cdot R+\frac{j(j+1)}{2(j-1)} \cdot \lambda\right]^{2}
\end{aligned}
$$

It follows that:

$$
\begin{aligned}
E\left(R_{i, j} R_{i^{\prime}, j}\right) & =E\left[\frac{j-1-\lambda j}{j-1} \cdot \bar{R}_{j}+\frac{j(j+1)}{2(j-1)} \cdot \lambda\right]^{2} \\
& =\left(\frac{j-1-\lambda j}{j-1}\right)^{2} E\left(\bar{R}_{j}{ }^{2}\right)+\frac{j-1-\lambda j}{j-1} \cdot \frac{j(j+1) \lambda}{2(j-1)} E\left(\bar{R}_{j}\right)+\frac{j^{2}(j+1)^{2} \lambda^{2}}{4(j-1)^{2}} .
\end{aligned}
$$

Substituting the standard results:

$$
E\left(\bar{R}_{j}\right)=\frac{j+1}{2}, \quad E\left(\bar{R}_{j}^{2}\right)=\frac{1}{j} \sum_{R=1}^{j} R^{2}=\frac{(2 j+1)(j+1)}{6},
$$

and rearranging terms, we obtain:

$$
E\left(R_{i, j} R_{i^{\prime}, j}\right)=\frac{(2 j+1)(j+1)}{6}-\frac{j(j+1)}{6} \lambda+\frac{(j+1) j^{2}}{12(j-1)} \lambda^{2} .
$$

Therefore:

$$
\begin{aligned}
\operatorname{Corr}\left(R_{i, j} R_{i, j}\right) & \equiv \frac{E\left(R_{i, j} R_{i, j}\right)-E\left(R_{i, j}\right) E\left(R_{i, j}\right)}{\sqrt{\operatorname{Var}\left(R_{i, j}\right) \operatorname{Var}\left(R_{i, j}\right)}} \\
& =\frac{12}{j^{2}-1} \cdot\left[\frac{(2 j+1)(j+1)}{6}-\frac{(j+1)^{2}}{4}-\frac{j(j+1)}{6} \lambda+\frac{(j+1) j^{2}}{12(j-1)} \lambda^{2}\right] \\
& =1-\frac{2 j}{j-1} \lambda+\frac{j^{2}}{(j-1)^{2}} \lambda^{2} \\
& =\left(1-\frac{j \lambda}{j-1}\right)^{2} .
\end{aligned}
$$

Substituting back $\lambda \equiv(1-\sqrt{\mu})(j-1) / j$ reduces the expression to:

$\operatorname{Corr}\left(R_{i, j}, R_{i^{\prime}, j}\right)=\mu$. 


\section{A.2. Sample Instructions for Experiment 1 (Committee Search with Uncorrelated Preferences), Majority Voting Rule Condition}

Welcome to our experiment on sequential observation and selection by committees. The instructions for the experiment are described below. If you follow them carefully and make good decisions, then you may earn $\$ 25$ or more for the session. In addition, you will earn a $\$ 5$ show-up bonus for your participation.

\section{Description of the Task}

Important business decisions are often made by committees whose members independently evaluate a number of alternatives in sequential manner. Because the committee members may come from different functional areas of the firm, they often use different criteria to evaluate the alternatives. For example, in evaluating the purchase of a new software product, the Accounting Manager might consider data security to be the highest concern, the Marketing Manager may consider ease of use to be more important, and the Finance Manager may be most concerned with how well the product integrates with existing software applications. Thus, as the committee of three members considers various software products, they may hold different opinions of each product considered. One product may rate high on data security (appealing to the Accounting Manager), but rate low on ease of use (unappealing to Marketing Manager). A second product alternative may rate high on ease of use and integration (appealing to both the Marketing and Finance Managers), but rate low on data security (unappealing to the Accounting Manager).

Together with two other participants, you will act as members of a 3-person committee. You will not be assigned to a specific managerial role (i.e., Accounting, Marketing, or Finance); rather, your evaluation for each alternative will be revealed to you in the alternative's relative rank (an explanation of relative and absolute ranks follows). Your objective is to try accepting as best an alternative as you can.

Your committee has to consider a maximum of 40 alternatives. Assume that these alternatives are ranked from 1 to 40 ("1" being the best) with no ties. We refer to these as absolute ranks. Absolute ranks are not revealed to the committee members until they reach a decision to accept a given alternative. Rather, as each alternative is presented, each committee member anonymously and independently makes an "accept" or "reject" decision which is based on the relative rank of the alternative. A relative rank is computed for each committee member separately in relation to the alternatives that the committee has already considered and rejected. Once rejected, an alternative cannot be recalled; if an alternative is accepted by the committee, further review of alternatives ends, the committee members' earnings for the trial are computed and revealed, and the game is repeated.

During this session, you will play 30 repeated games of this sequential observation and selection task, and will always be playing with the same 3-person committee. Each game is identical except that the 
alternatives that you consider appear in a different random order of ranks. And, as the example above with the Accounting, Marketing, and Finance managers shows, each member of your 3-person committee will have different relative and absolute ranks for the alternatives presented.

\section{Decision Rules of the Committee}

In the decision task described above, whether an alternative is adopted depends not only on how each committee member evaluates the alternative, but also on the decision rule used by the committee. For 3person committees, several decision rules - Minority, Majority, and Unanimity may be considered.

Minority: under the minority rule, the current alternative is adopted if one or more committee members decide to accept it.

Majority: under the majority rule, the current alternative is adopted if two or more members decide to accept it.

Unanimity: under the unanimity rule, the current alternative is adopted if all three members agree to accept it.

In the present experiment, you and the other two members of your committee will be using the Majority rule in deciding between alternatives. Thus, two or more members of the committee must decide to "accept" before the alternative is adopted.

\section{Determining Your Payoff}

Your payoff (in points) in each game will be determined by the following formula:

Payoff $=30-f$,

where $f$ is the absolute rank of the alternative among all 40 alternatives (including the ones that remain to be considered).

For example, supposing that your committee has reviewed and rejected 24 (out of 40) alternatives. As you consider the $25^{\text {th }}$ alternative, you notice that it is currently ranked $2^{\text {nd }}$ among all the alternatives considered so far. Assume that you and at least one of the other committee members decide to accept this alternative. With two accept decisions, the "majority rule" is satisfied and the alternative is adopted; a further review of alternatives ends, and the absolute ranks of all the 40 alternatives are revealed. In this example, the absolute rank of the alternative (according to your criterion) that the committee accepted turns out to be $f=4$, as two of the fifteen remaining alternatives that have not been considered are ranked higher on your criterion. Your earnings for this game are computed to be $(30-4)=26$ points. The earnings of the other members are determined in a similar way, using their own absolute ranks of alternative. 
To further illustrate the kind of decisions that you will be making in the game, and to highlight the two different notions of relative rank and absolute rank, consider the following examples which - to simplify matters - assume that a maximum of 10 alternatives to be considered.

\section{Relative and Absolute Ranks - Example \#1}

When the game begins, the relative rank of the first alternative is always 1 for each committee member, indicating that (by definition) this alternative is the best of those observed thus far. The relative ranks of the remaining alternatives are unknown at this point (see table below).

\begin{tabular}{|l|l|l|l|l|l|l|l|l|l|l|}
\hline Alternative \# & 1 & 2 & 3 & 4 & 5 & 6 & 7 & 8 & 9 & 10 \\
\hline Relative Rank & 1 & - & - & - & - & - & - & - & - & - \\
\hline
\end{tabular}

Assume that the committee decides to reject the first alternative. Then, the second alternative is presented and the relative ranks of the first two alternatives are compared to each other. Note that the relative rank of any alternative may change as each new alternative is considered. In this example (see table below) you find that alternative \#2 is better than alternative \#1 (according to your criterion). Note, too, that you are only aware of the relative ranks for your criterion, not those of the other committee members.

\begin{tabular}{|l|l|l|l|l|l|l|l|l|l|l|}
\hline Alternative \# & $\mathbf{1}$ & $\mathbf{2}$ & $\mathbf{3}$ & $\mathbf{4}$ & $\mathbf{5}$ & $\mathbf{6}$ & $\mathbf{7}$ & $\mathbf{8}$ & $\mathbf{9}$ & $\mathbf{1 0}$ \\
\hline Relative Rank & 2 & 1 & - & - & - & - & - & - & - & - \\
\hline
\end{tabular}

Suppose that the committee decides to reject alternative \#2. The relative ranks of the first three alternatives are now presented (see table below).

\begin{tabular}{|l|l|l|l|l|l|l|l|l|l|l|}
\hline Alternative \# & $\mathbf{1}$ & $\mathbf{2}$ & $\mathbf{3}$ & $\mathbf{4}$ & $\mathbf{5}$ & $\mathbf{6}$ & $\mathbf{7}$ & $\mathbf{8}$ & $\mathbf{9}$ & $\mathbf{1 0}$ \\
\hline Relative Rank & 2 & 1 & 3 & - & - & - & - & - & - & - \\
\hline
\end{tabular}

Once again, the committee decides to reject this alternative and subsequently considers alternative \#4 (see table below).

\begin{tabular}{|l|l|l|l|l|l|l|l|l|l|l|}
\hline Alternative \# & $\mathbf{1}$ & $\mathbf{2}$ & $\mathbf{3}$ & $\mathbf{4}$ & $\mathbf{5}$ & $\mathbf{6}$ & $\mathbf{7}$ & $\mathbf{8}$ & $\mathbf{9}$ & $\mathbf{1 0}$ \\
\hline Relative Rank & 3 & 1 & 4 & 2 & - & - & - & - & - & - \\
\hline
\end{tabular}

Alternative \#4 is now ranked as the $2^{\text {nd }}$ best alternative (according to your criterion) of the alternatives considered so far. Let's assume that you and at least one other committee member decide to accept this alternative. Further alternatives are not considered, the game ends, and the absolute ranks of all 10 alternatives are revealed in the table below: 


\begin{tabular}{|l|l|l|l|l|l|l|l|l|l|l|}
\hline Alternative \# & $\mathbf{1}$ & $\mathbf{2}$ & $\mathbf{3}$ & $\mathbf{4}$ & $\mathbf{5}$ & $\mathbf{6}$ & $\mathbf{7}$ & $\mathbf{8}$ & $\mathbf{9}$ & $\mathbf{1 0}$ \\
\hline Absolute rank & 5 & 2 & 8 & 3 & 7 & 1 & 4 & 10 & 9 & 6 \\
\hline
\end{tabular}

The table above shows that the absolute rank of alternative \#4 is 3 , but when the committee first accepted this alternative, it was ranked $2^{\text {nd }}$ among the first 4 considered. It turns out that one of the remaining alternatives (\#6) was ranked higher. Your payoff for the game is $(30-3)=27$ points.

\section{Relative and Absolute Ranks - Example \#2}

Suppose that in this example the committee has rejected the first eight alternatives. Alternative \#9 is considered, with the following relative ranks:

\begin{tabular}{|l|l|l|l|l|l|l|l|l|l|l|}
\hline Alternative \# & $\mathbf{1}$ & $\mathbf{2}$ & $\mathbf{3}$ & $\mathbf{4}$ & $\mathbf{5}$ & $\mathbf{6}$ & $\mathbf{7}$ & $\mathbf{8}$ & $\mathbf{9}$ & $\mathbf{1 0}$ \\
\hline Absolute rank & 4 & 1 & 7 & 2 & 9 & 3 & 6 & 8 & 5 & - \\
\hline
\end{tabular}

Your choice is either to accept alternative \#9, with a relative rank of 5 on your criterion, or reject this alternative and consider the tenth and final alternative, which must be accepted. Again, assume that the committee rejects alternative \#9. Alternative \#10 is observed, and the following absolute ranks are revealed:

\begin{tabular}{|l|l|l|l|l|l|l|l|l|l|l|}
\hline Alternative \# & $\mathbf{1}$ & $\mathbf{2}$ & $\mathbf{3}$ & $\mathbf{4}$ & $\mathbf{5}$ & $\mathbf{6}$ & $\mathbf{7}$ & $\mathbf{8}$ & $\mathbf{9}$ & $\mathbf{1 0}$ \\
\hline Absolute rank & 5 & 1 & 8 & 2 & 10 & 3 & 7 & 9 & 6 & 4 \\
\hline
\end{tabular}

Because this (last) alternative must be accepted, your payoff for this game is $(30-4)=26$ points. The payoffs for other committee members depend on how this alternative ranks on their criteria.

These two examples illustrate that:

- Relative ranks may change as each new alternative is considered.

- Members of your 3-person committee will have different relative and absolute ranks for the alternatives presented.

- Each committee member may decide to either accept or reject an alternative, but members are required to accept the final (\#40) alternative.

- Two or more members of the committee must decide to "accept" before the alternative is adopted.

- Alternatives are observed in a different random order of ranking in each game.

- Members of the committee are the same in each of the 30 games.

- Your earnings for each game are determined by the formula: $30-f$, where $f$ is the absolute rank of the alternative the committee accepts. Note that if the absolute rank of the alternative is greater than 30 , you could lose money for that game. 
After all the participants have completed reading these instructions, the experimenter will be happy to answer any questions that you may have. You may take notes on scratch paper that we provide. If you have questions, please raise your hand.

\section{Determining Your Payment at the End of the Session}

You will be paid in cash at the end of the session: Five of the 30 games that you have completed (same for

all the participants) will be chosen randomly, and your cumulative earnings in these five games will be converted to dollars at the rate of $\mathbf{\$ 0 . 2 0}$ per point. In addition, you will be paid a $\$ 5$ show up fee.

Thank you for your participation and good luck! 


\section{A.3. Sample Instructions and Post-Experimental Questionnaire for Experiment 2 (Committee Search with Perfectly Correlated Preferences), Majority Voting Rule Condition}

Welcome to our experiment on sequential observation and selection by committees. The instructions for the experiment are described below. If you follow them carefully and make good decisions, then you may earn $\$ 25$ or more for the session. In addition, you will earn a $\$ 5$ show-up bonus for your participation.

\section{Description of the Task}

Important business decisions are often made by committees whose members independently evaluate a number of alternatives in sequential manner. Together with two other participants in the computer lab, you will act as members of a 3-person committee. You will review alternatives sequentially, but the only information you have about each alternative is the relative rank (an explanation of relative and absolute ranks follows). Your objective is to try to accept an alternative with the best (lowest) absolute rank.

Your committee has to consider a maximum of 40 alternatives. Assume that these alternatives are ranked from 1 to 40 ("1" being the best) with no ties. We refer to these as absolute ranks. Absolute ranks are not revealed to the committee members until they reach a decision to accept a given alternative. Rather, as each alternative is presented, each committee member anonymously and independently makes an "accept" or "reject" decision which is based on the relative rank of the alternative. A relative rank is the rank of the alternative compared to those seen thus far. Each committee member will see the same relative rank for each alternative, but may make a different accept or reject decision. Once rejected, an alternative cannot be recalled; if an alternative is accepted by the committee, further review of alternatives ends, the committee members' earnings for the trial are computed and revealed, and the game is repeated.

During this session, you will play 30 repeated games of this sequential observation and selection task, and will always be playing with the same three-person committee. Each game is identical except that the alternatives that you consider appear in a different random order of ranks.

\section{Decision Rules of the Committee}

In the decision task described above, whether an alternative is adopted depends on how many "accept" decisions the committee makes for an alternative, and the decision rule used by the committee. For 3-person committees, several decision rules - Minority, Majority, and Unanimous may be considered.

Minority: under the minority rule, the current alternative is adopted if one or more committee members decide to accept it.

Majority: under the majority rule, the current alternative is adopted if two or more members decide to accept it. 
Unanimous: under the unanimous rule, the current alternative is adopted if all three members agree to accept it.

In the present experiment, you and the other two members of your committee will be using the Majority rule in deciding between alternatives. Thus, two or more members of the committee must decide to "accept" before the alternative is adopted.

\section{Determining Your Payoff}

Your payoff (in points) in each game will be determined by the following formula:

Payoff $=30-f$,

where $f$ is the absolute rank of the alternative among all 40 alternatives (including the ones that remain to be considered).

For example, supposing that your committee has reviewed and rejected 24 (out of 40) alternatives. As you consider the $25^{\text {th }}$ alternative, you notice that it is currently ranked $2^{\text {nd }}$ among all the alternatives considered so far. Assume that you and at least one of the other committee members decide to accept this alternative. With two accept decisions, the "majority rule" is satisfied and the alternative is adopted; a further review of alternatives ends, and the absolute ranks of all the 40 alternatives are revealed. In this example, the absolute rank of the alternative that your committee accepted turns out to be $f=4$, as two of the fifteen remaining alternatives that have not been considered are ranked higher. Your earnings (and the earnings of the other two committee members) for this game are computed to be $(30-4)=26$ points.

To further illustrate the kind of decisions that you will be making in the game, and to highlight the two different notions of relative rank and absolute rank, consider the following examples which - to simplify matters - assume that a maximum of 10 alternatives to be considered.

\section{Relative and Absolute Ranks - Example \#1}

When the game begins, the relative rank of the first alternative is always 1 for each committee member, indicating that (by definition) this alternative is the best of those observed thus far. The relative ranks of the remaining alternatives are unknown at this point (see table below).

\begin{tabular}{|l|l|l|l|l|l|l|l|l|l|l|}
\hline Alternative \# & 1 & 2 & 3 & 4 & 5 & 6 & 7 & 8 & 9 & 10 \\
\hline Relative Rank & 1 & - & - & - & - & - & - & - & - & - \\
\hline
\end{tabular}

Assume that the committee decides to reject the first alternative. Then, the second alternative is presented and the relative ranks of the first two alternatives are compared to each other. Note that the relative rank of any alternative may change as each new alternative is considered. In this example (see table below) you find that alternative \#2 is better than alternative \#1 (according to your criterion). 


\begin{tabular}{|l|l|l|l|l|l|l|l|l|l|l|}
\hline Alternative \# & $\mathbf{1}$ & $\mathbf{2}$ & $\mathbf{3}$ & $\mathbf{4}$ & $\mathbf{5}$ & $\mathbf{6}$ & $\mathbf{7}$ & $\mathbf{8}$ & $\mathbf{9}$ & $\mathbf{1 0}$ \\
\hline Relative Rank & 2 & 1 & - & - & - & - & - & - & - & - \\
\hline
\end{tabular}

Suppose that the committee decides to reject alternative \#2. The relative ranks of the first three alternatives are now presented (see table below).

\begin{tabular}{|l|l|l|l|l|l|l|l|l|l|l|}
\hline Alternative \# & $\mathbf{1}$ & $\mathbf{2}$ & $\mathbf{3}$ & $\mathbf{4}$ & $\mathbf{5}$ & $\mathbf{6}$ & $\mathbf{7}$ & $\mathbf{8}$ & $\mathbf{9}$ & $\mathbf{1 0}$ \\
\hline Relative Rank & 2 & 1 & 3 & - & - & - & - & - & - & - \\
\hline
\end{tabular}

Once again, the committee decides to reject this alternative and subsequently considers alternative \#4 (see table below).

\begin{tabular}{|l|l|l|l|l|l|l|l|l|l|l|}
\hline Alternative \# & $\mathbf{1}$ & $\mathbf{2}$ & $\mathbf{3}$ & $\mathbf{4}$ & $\mathbf{5}$ & $\mathbf{6}$ & $\mathbf{7}$ & $\mathbf{8}$ & $\mathbf{9}$ & $\mathbf{1 0}$ \\
\hline Relative Rank & 3 & 1 & 4 & 2 & - & - & - & - & - & - \\
\hline
\end{tabular}

Alternative \#4 is now ranked as the $2^{\text {nd }}$ best alternative (according to your criterion) of the alternatives considered so far. Let's assume that you and at least one other committee member decide to accept this alternative. Further alternatives are not considered, the game ends, and the absolute ranks of all 10 alternatives are revealed in the table below:

\begin{tabular}{|l|l|l|l|l|l|l|l|l|l|l|}
\hline Alternative \# & $\mathbf{1}$ & $\mathbf{2}$ & $\mathbf{3}$ & $\mathbf{4}$ & $\mathbf{5}$ & $\mathbf{6}$ & $\mathbf{7}$ & $\mathbf{8}$ & $\mathbf{9}$ & $\mathbf{1 0}$ \\
\hline Final Rank & 5 & 2 & 8 & 3 & 7 & 1 & 4 & 10 & 9 & 6 \\
\hline
\end{tabular}

The table above shows that the absolute rank of alternative \#4 is 3 , but when the committee first accepted this alternative, it was ranked $2^{\text {nd }}$ among the first 4 considered. It turns out that one of the remaining alternatives (\#6) was ranked higher. Your payoff for the game is $(30-3)=27$ points.

\section{Relative and Absolute Ranks - Example \#2}

Suppose that in this example the committee has rejected the first eight alternatives. Alternative \#9 is considered, with the following relative ranks:

\begin{tabular}{|l|l|l|l|l|l|l|l|l|l|l|}
\hline Alternative \# & $\mathbf{1}$ & $\mathbf{2}$ & $\mathbf{3}$ & $\mathbf{4}$ & $\mathbf{5}$ & $\mathbf{6}$ & $\mathbf{7}$ & $\mathbf{8}$ & $\mathbf{9}$ & $\mathbf{1 0}$ \\
\hline Final Rank & 4 & 1 & 7 & 2 & 9 & 3 & 6 & 8 & 5 & - \\
\hline
\end{tabular}

Your choice is either to accept alternative \#9, with a relative rank of 5 on your criterion, or reject this alternative and consider the tenth and final alternative, which must be accepted. Again, assume that the committee rejects alternative \#9. Alternative \#10 is observed, and the following absolute ranks are revealed: 


\begin{tabular}{|l|l|l|l|l|l|l|l|l|l|l|}
\hline Alternative \# & $\mathbf{1}$ & $\mathbf{2}$ & $\mathbf{3}$ & $\mathbf{4}$ & $\mathbf{5}$ & $\mathbf{6}$ & $\mathbf{7}$ & $\mathbf{8}$ & $\mathbf{9}$ & $\mathbf{1 0}$ \\
\hline Final Rank & 5 & 1 & 8 & 2 & 10 & 3 & 7 & 9 & 6 & 4 \\
\hline
\end{tabular}

Because this (last) alternative must be accepted, your payoff for this game is $(30-4)=26$ points. The payoff for each committee member is identical.

These two examples illustrate that:

- Relative ranks may change as each new alternative is considered.

- Members of your 3-person committee will have identical relative and absolute ranks for the alternatives presented.

- Each committee member may decide to either accept or reject an alternative, but members are required to accept the final (\#40) alternative.

- Two or more members of the committee must decide to "accept" before the alternative is adopted.

- Alternatives are observed in a different random order of ranking in each game.

- Members of the committee are the same in each of the 30 games.

- Your earnings for each game are determined by the formula: $30-f$, where $f$ is the absolute rank of the alternative the committee accepts. Note that if the final rank of the alternative is greater than 30 , you could lose money for that game (negative values appear in parenthesis).

After all the participants have completed reading these instructions, the experimenter will be happy to answer any questions that you may have. You may take notes on scratch paper that we provide. If you have questions, please raise your hand.

\section{Determining Your Payment at the End of the Session}

You will be paid in cash at the end of the session: Five of the 30 games that you have completed (same for all the participants) will be chosen randomly, and your cumulative earnings in these five games will be converted to dollars at the rate of $\mathbf{\$ 0 . 2 0}$ per point. In addition, you will be paid a $\$ 5$ show up fee.

When the experiment ends, please raise your hand and the supervisor will come to assist you.

Thank you for your participation and good luck! 


\section{Post-Experimental Questionnaire}

Please answer the following $\mathbf{5}$ questions regarding the study you just completed. You'll be rewarded $\$ 1$ for every correctly answered question.

No.

Date

1. What is the maximum number of alternatives that you might observe during a given game? (please circle)
a. 10
b. 20
c. 30
d. 40

2. Supposing that your committee has already observed and rejected the first three alternatives whose absolute ranks are 5,15 , and 25 , respectively. The next $\left(4^{\text {th }}\right)$ alternative has an absolute rank of 8 . What is its relative rank? (please circle)
a. 1
b. 2
c. 3
d. 4

3. In the scenario described in Question 2, if your committee accepts the $4^{\text {th }}$ alternative which has an absolute rank of 8 , what will be your payoff in the game (in points)? (please circle)
a. 20
b. 22
c. 26
d. 28

4. Supposing that your committee has already observed and rejected the first three alternatives whose absolute ranks are 5,15 , and 25 , respectively. The next $\left(4^{\text {th }}\right)$ alternative has a relative rank of 3 . What are its possible absolute ranks? (please circle)
a. Between 1 and 4
b. Between 6 and 14
c. Between 16 and 24
d. Between 26 and 40 
5. Supposing that your committee has already observed and rejected the first three alternatives. The next $\left(4^{\text {th }}\right)$ alternative has a relative rank of 2 . Under which (one or more) of the following scenarios will the alternative be accepted by the committee? (please circle ALL that apply)

a. Only one of the three members votes ACCEPT

b. Two of the three members vote ACCEPT while the third member votes REJECT

c. All three members vote ACCEPT

Thank you for your answers.

(The correct answers are: $1-d ; 2-b ; 3-b ; 4-c ; 5-a, b$, and $c$ if participant was in the minority rule condition, $b$ and $c$ if participant was in the majority rule condition, $c$ if participant was in the unanimity rule condition ) 


\section{A.4. Sample Instructions for Experiment 3 (Single-Decision Maker Search)}

Welcome to our experiment on sequential observation and selection. The instructions for the experiment are described below. If you follow them carefully and make good decisions, then you may earn $\$ 25$ or more for the session. In addition, you will earn a $\$ 5$ show-up bonus for your participation.

\section{Description of the Task}

Important business decisions are often made by reviewing alternatives in sequential fashion, and making accept or reject decisions as each alternative is presented. In this experiment you are asked to consider a maximum of 40 alternatives. Assume that these alternatives are ranked from 1 to 40 (" 1 " being the best) with no ties. We refer to these as absolute ranks. Absolute ranks are not revealed to you until you decide to accept a given alternative. Rather, as each alternative is presented, you will see the relative rank of each alternative and make an "accept" or "reject" decision based on the relative rank of the alternative. A relative rank is the rank of the alternative compared to those seen thus far. Once rejected, an alternative cannot be recalled; if an alternative is accepted, further review of alternatives ends, and the computer displays the absolute ranks for all 40 alternatives and your earnings for the trial.

During this session, you will play 50 repeated games of this sequential observation and selection task. Each game is identical except that the alternatives that you consider appear in a different random order of ranks.

\section{Determining Your Payoff}

Your payoff (in \$US) in each game will be determined by the following formula:

$$
\text { Payoff }=9-f,
$$

where $f$ is the absolute rank of the alternative among all 40 alternatives (including the ones that remain to be considered) that you have selected.

For example, suppose that you have reviewed and rejected 24 (out of 40) alternatives. As you consider the $25^{\text {th }}$ alternative, you notice that it is currently ranked $2^{\text {nd }}$ (relative rank) among all the alternatives considered so far. Assume that you decide to accept this alternative. Further review of alternatives ends, and the absolute ranks of all the 40 alternatives are revealed. In this example, the absolute rank of the alternative that you accepted turns out to be $f=4$, as two of the fifteen remaining alternatives that have not been considered are ranked higher. Your earnings for this game are computed to be $(9-4)=$ $\$ 5$. 
To further illustrate the kind of decisions that you will be making in the game, and to highlight the two different notions of relative rank and absolute rank, consider the following examples which - to simplify matters - assume a maximum of 10 alternatives to be considered.

\section{Relative and Absolute Ranks - Example \#1}

When the game begins, the relative rank of the first alternative is always 1 , indicating that (by definition) this alternative is the best of those observed thus far. The relative ranks of the remaining alternatives are unknown at this point (see table below).

\begin{tabular}{|l|l|l|l|l|l|l|l|l|l|l|}
\hline Alternative \# & 1 & 2 & 3 & 4 & 5 & 6 & 7 & 8 & 9 & 10 \\
\hline Relative Rank & 1 & - & - & - & - & - & - & - & - & - \\
\hline
\end{tabular}

Assume that you decide to reject the first alternative. Then, the second alternative is presented and the relative ranks of the first two alternatives are compared to each other. Note that the relative rank of any alternative may change as each new alternative is considered. In this example (see table below) you find that alternative \#2 is better than alternative \#1.

\begin{tabular}{|l|l|l|l|l|l|l|l|l|l|l|}
\hline Alternative \# & $\mathbf{1}$ & $\mathbf{2}$ & $\mathbf{3}$ & $\mathbf{4}$ & $\mathbf{5}$ & $\mathbf{6}$ & $\mathbf{7}$ & $\mathbf{8}$ & $\mathbf{9}$ & $\mathbf{1 0}$ \\
\hline Relative Rank & 2 & 1 & - & - & - & - & - & - & - & - \\
\hline
\end{tabular}

Suppose you decide to reject alternative \#2. The relative ranks of the first three alternatives are now presented (see table below).

\begin{tabular}{|l|l|l|l|l|l|l|l|l|l|l|}
\hline Alternative \# & $\mathbf{1}$ & $\mathbf{2}$ & $\mathbf{3}$ & $\mathbf{4}$ & $\mathbf{5}$ & $\mathbf{6}$ & $\mathbf{7}$ & $\mathbf{8}$ & $\mathbf{9}$ & $\mathbf{1 0}$ \\
\hline Relative Rank & 2 & 1 & 3 & - & - & - & - & - & - & - \\
\hline
\end{tabular}

Once again, you decide to reject this alternative and subsequently considers alternative \#4 (see table below).

\begin{tabular}{|l|l|l|l|l|l|l|l|l|l|l|}
\hline Alternative \# & $\mathbf{1}$ & $\mathbf{2}$ & $\mathbf{3}$ & $\mathbf{4}$ & $\mathbf{5}$ & $\mathbf{6}$ & $\mathbf{7}$ & $\mathbf{8}$ & $\mathbf{9}$ & $\mathbf{1 0}$ \\
\hline Relative Rank & 3 & 1 & 4 & 2 & - & - & - & - & - & - \\
\hline
\end{tabular}


Alternative \#4 is now ranked as the $2^{\text {nd }}$ best alternative of the alternatives considered so far. Let's assume that you decide to accept this alternative. Further alternatives are not considered, the game ends, and the absolute ranks of all 10 alternatives are revealed in the table below:

\begin{tabular}{|l|l|l|l|l|l|l|l|l|l|l|}
\hline Alternative \# & $\mathbf{1}$ & $\mathbf{2}$ & $\mathbf{3}$ & $\mathbf{4}$ & $\mathbf{5}$ & $\mathbf{6}$ & $\mathbf{7}$ & $\mathbf{8}$ & $\mathbf{9}$ & $\mathbf{1 0}$ \\
\hline Absolute Rank & 5 & 2 & 8 & 3 & 7 & 1 & 4 & 10 & 9 & 6 \\
\hline
\end{tabular}

The table above shows that the absolute rank of alternative \#4 is 3 , but when you accepted this alternative, it was ranked $2^{\text {nd }}$ among the first 4 considered. It turns out that one of the remaining alternatives (\#6) was ranked higher. Your payoff for the game is $(9-3)=\$ 6$.

\section{Relative and Absolute Ranks - Example \#2}

Suppose that in this example you have rejected the first eight alternatives. Alternative \#9 is considered, with the following relative ranks:

\begin{tabular}{|l|l|l|l|l|l|l|l|l|l|l|}
\hline Alternative \# & $\mathbf{1}$ & $\mathbf{2}$ & $\mathbf{3}$ & $\mathbf{4}$ & $\mathbf{5}$ & $\mathbf{6}$ & $\mathbf{7}$ & $\mathbf{8}$ & $\mathbf{9}$ & $\mathbf{1 0}$ \\
\hline Relative Rank & 4 & 1 & 7 & 2 & 9 & 3 & 6 & 8 & 5 & - \\
\hline
\end{tabular}

Your choice is either to accept alternative \#9, with a relative rank of 5, or reject this alternative and consider the tenth and final applicant, which must be accepted. Again, assume that you reject alternative \#9. Alternative \#10 is observed, and the following absolute ranks are revealed:

\begin{tabular}{|l|l|l|l|l|l|l|l|l|l|l|}
\hline Alternative \# & $\mathbf{1}$ & $\mathbf{2}$ & $\mathbf{3}$ & $\mathbf{4}$ & $\mathbf{5}$ & $\mathbf{6}$ & $\mathbf{7}$ & $\mathbf{8}$ & $\mathbf{9}$ & $\mathbf{1 0}$ \\
\hline Final Rank & 5 & 1 & 8 & 2 & 10 & 3 & 7 & 9 & 6 & 4 \\
\hline
\end{tabular}

Because this (last) applicant must be accepted, your payoff for this game is $(9-4)=\$ 5$.

These two examples illustrate that:

- Relative ranks may change as each new alternative is considered.

- You may decide to either accept or reject an alternative, but you are required to accept the final (\#40) alternative.

- Alternatives are observed in a different random order of ranking in each game.

- Your earnings for each game are determined by the formula: $9-f$, where $f$ is the absolute rank of the alternative you accepted. Note that if the final rank of the alternative is greater than 9 you could lose money for that game. 
After all the participants have completed reading these instructions, the experimenter will be happy to answer any questions that you may have. You may take notes on scratch paper that we provide. If you have questions, please raise your hand. When everyone is ready, the experimenter will reveal the "Passcode", which you will enter on the computer screen to initiate the software program.

\section{Determining Your Payment at the End of the Session}

You will be paid in cash at the end of the session: Five of the 50 games that you have completed will be chosen randomly, and your total earnings in each of these five games will be added together. In addition, you will be paid a $\$ 5$ show up bonus.

Thank you for your participation and good luck! 
A.5. Search Performance in Experiments 1, 2, and 3

(a) Experiment 1 (committee search with uncorrelated preferences)

$\underline{\text { Minority rule condition }}$

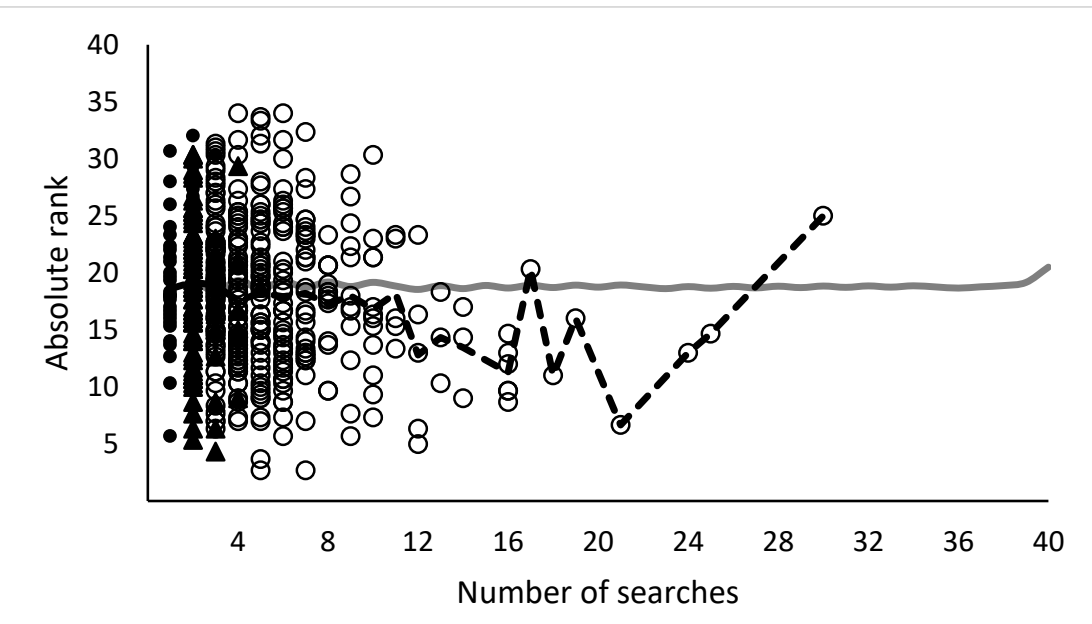

Majority rule condition

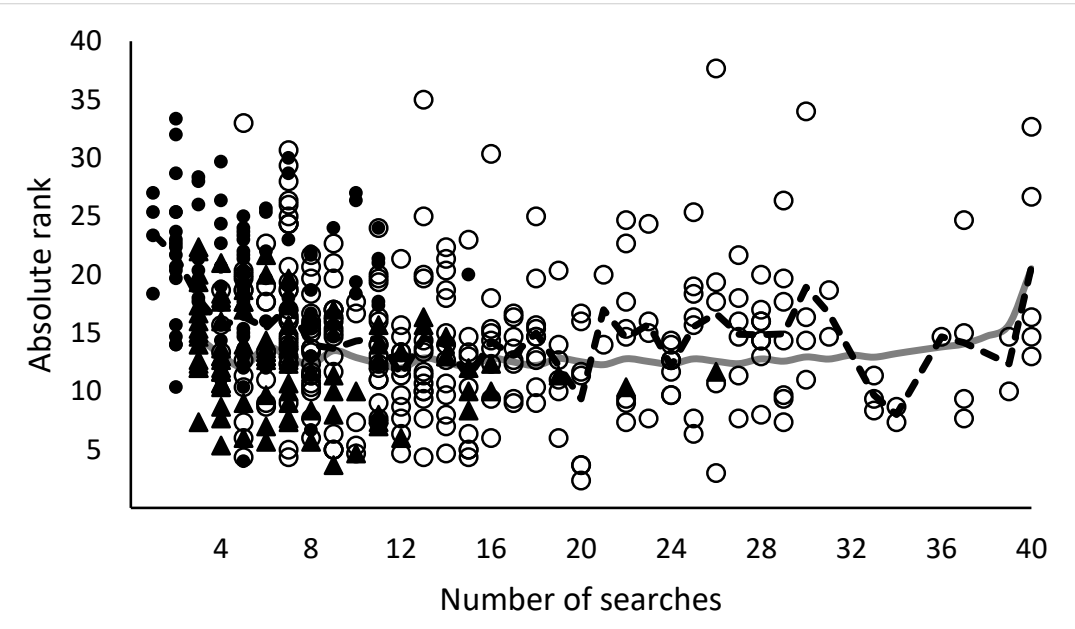


(a) Experiment 1 (committee search with uncorrelated preferences) (cont'd)

\section{Unanimity rule condition}

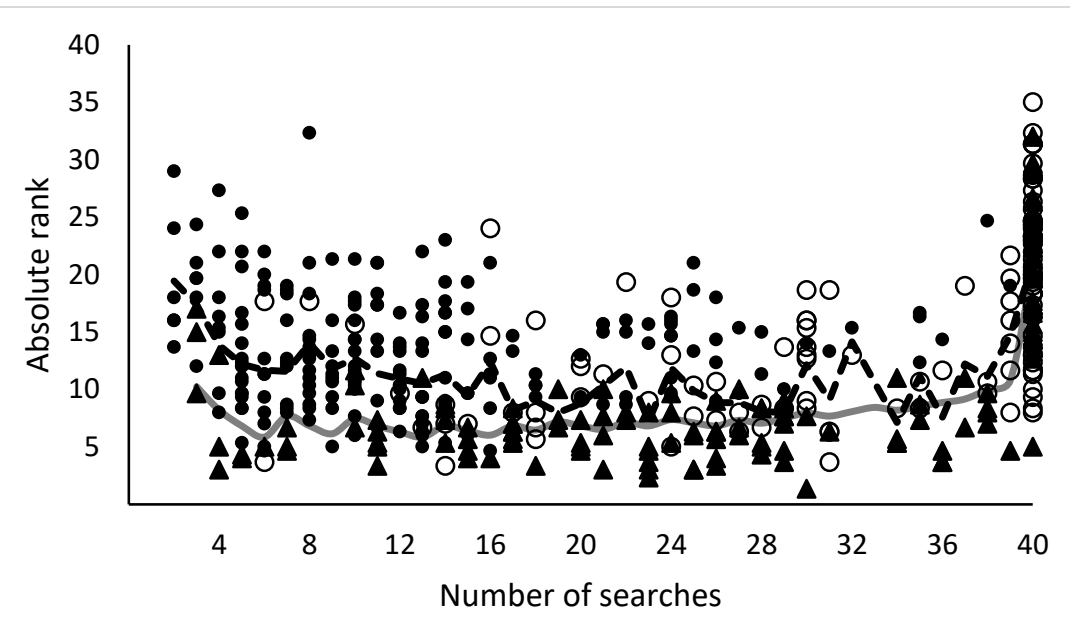

(b) Experiment 2 (committee search with perfectly correlated preferences)

Minority rule condition

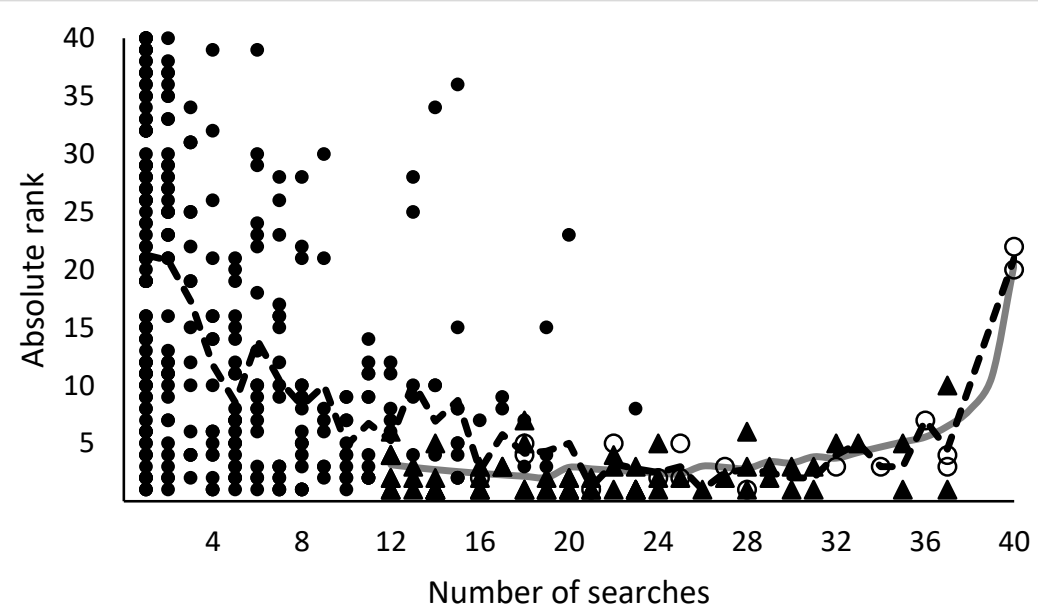


(b) Experiment 2 (committee search with perfectly correlated preferences) (cont'd)

Majority rule condition

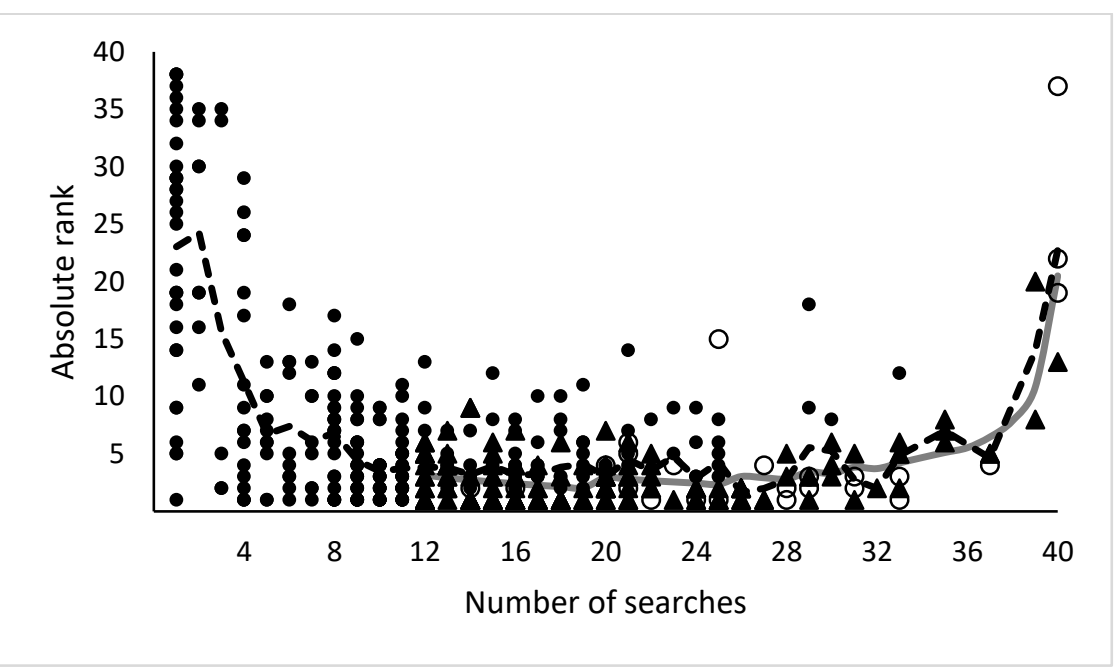

$\underline{\text { Unanimity rule condition }}$

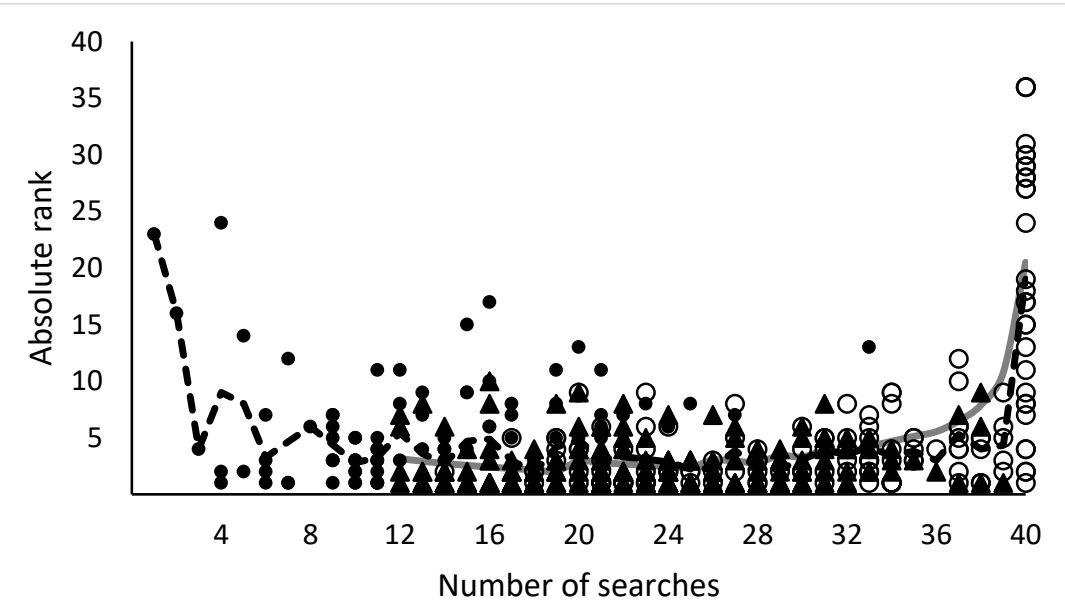


(b) Experiment 3 (single-DM search)

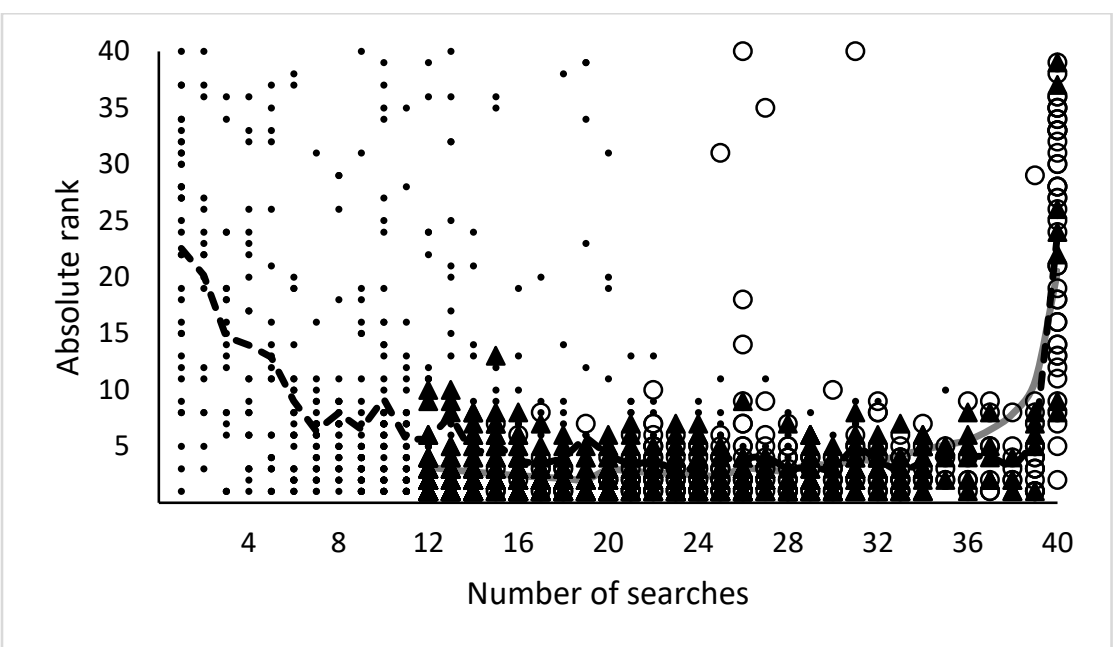

Note. Each marker represents a single search game. In each panel, dot (•) and hollow (०) markers indicate under- and over-search, respectively, when compared with the equilibrium strategy; while triangular ( $\mathbf{A})$ markers indicate that the final decision making unit (three-person committee in Experiment 1 and 2, single DM in Experiment 3) had made exactly as many searches in that game as in equilibrium. The dashed line indicates observed mean absolute ranks across final decision making units by number of searches; the gray line indicates the corresponding expected absolute ranks under the equilibrium strategy. 
A.6. Game-level Deviation Counts in Experiments 1, 2, and 3

\begin{tabular}{|c|c|c|c|c|c|c|c|c|c|}
\hline & \multicolumn{3}{|c|}{ Minority rule $(q=1)$} & \multicolumn{3}{|c|}{ Majority rule $(q=2)$} & \multicolumn{3}{|c|}{ Unanimity rule $(q=3)$} \\
\hline & \multicolumn{9}{|c|}{ No. of games with (relative to equilibrium play) ... } \\
\hline & & No & & & $\mathrm{No}$ & & & $\mathrm{No}$ & \\
\hline & Under-search & deviation & Over-search & Under-search & deviation & Over-search & Under-search & deviation & Over-search \\
\hline Expt. 1 & $2.1(4.2)$ & $6.3(4.7)$ & $21.7(6.5)$ & $6.4(6.2)$ & $6.1(4.3)$ & $17.4(7.8)$ & $13.4(7.3)$ & $9.2(5.4)$ & $7.4(5.0)$ \\
\hline Expt. 2 & $24.1(8.9)$ & $4.7(6.9)$ & $1.2(2.4)$ & $18.1(7.2)$ & $10.2(5.6)$ & $1.7(2.6)$ & $6.1(5.8)$ & $13.6(5.7)$ & $10.3(4.5)$ \\
\hline Expt. 3 & & & & $19.4(13.8)$ & $21.7(10.4)$ & $8.9(7.4)$ & & & \\
\hline
\end{tabular}

Note. SDs (with committee as the unit of analysis in Experiment 1 and 2 and individual subject as the unit of analysis in Experiment 3) are in parentheses. The sum of the three means in any condition is (up to rounding errors) equal to the total number of games per session, that is, 30 in Experiments 1 and 2, and 50 in Experiment 3. 
A.7 Empirical Best Response Threshold Ranks and Comparisons with Equilibrium

\begin{tabular}{|c|c|c|c|c|c|c|c|c|c|c|c|c|}
\hline \multirow{3}{*}{$\begin{array}{l}j \\
1\end{array}$} & \multicolumn{6}{|c|}{ Experiment 1 (uncorrelated preferences) } & \multicolumn{6}{|c|}{ Experiment 2 (perfectly correlated preferences) } \\
\hline & \multicolumn{2}{|c|}{$q=1$} & \multicolumn{2}{|c|}{$q=2$} & \multicolumn{2}{|c|}{$q=3$} & \multicolumn{2}{|c|}{$q=1$} & \multicolumn{2}{|c|}{$q=2$} & \multicolumn{2}{|c|}{$q=3$} \\
\hline & 0 & 0 & 0 & 0 & 0 & 0 & 0 & 0 & 0 & 0 & 0 & 0 \\
\hline 2 & 1 & 0 & 1 & 1 & 0 & 0 & 0 & 0 & 0 & 0 & 0 & 0 \\
\hline 3 & 1 & 0 & 1 & 0 & 1 & 0 & 0 & 0 & 0 & 0 & 0 & 0 \\
\hline 4 & 2 & 0 & 1 & 0 & 1 & 0 & 1 & 1 & 0 & 0 & 0 & 0 \\
\hline 5 & 2 & 0 & 2 & 1 & 1 & 0 & 1 & 1 & 0 & 0 & 0 & 0 \\
\hline 6 & 3 & 0 & 2 & 0 & 2 & 1 & 1 & 1 & 0 & 0 & 0 & 0 \\
\hline 7 & 3 & 0 & 2 & 0 & 2 & 0 & 1 & 1 & 0 & 0 & 0 & 0 \\
\hline 8 & 3 & -1 & 3 & 1 & 2 & 0 & 1 & 1 & 0 & 0 & 1 & 1 \\
\hline 9 & 3 & -1 & 3 & 0 & 3 & 1 & 1 & 1 & 1 & 1 & 1 & 1 \\
\hline 10 & 3 & -2 & 3 & 0 & 3 & 0 & 2 & 2 & 1 & 1 & 1 & 1 \\
\hline 11 & 3 & -2 & 4 & 1 & 3 & 0 & 2 & 2 & 1 & 1 & 1 & 1 \\
\hline 12 & 4 & -1 & 4 & 1 & 4 & 1 & 2 & 1 & 1 & 0 & 1 & 0 \\
\hline 13 & 4 & -2 & 4 & 0 & 4 & 1 & 1 & 0 & 1 & 0 & 1 & 0 \\
\hline 14 & 4 & -2 & 5 & 1 & 5 & 1 & 2 & 1 & 1 & 0 & 1 & 0 \\
\hline 15 & 5 & -2 & 5 & 1 & 5 & 1 & 2 & 1 & 1 & 0 & 1 & 0 \\
\hline 16 & 6 & -1 & 6 & 1 & 5 & 1 & 2 & 1 & 1 & 0 & 2 & 1 \\
\hline 17 & 6 & -2 & 6 & 1 & 6 & 1 & 2 & 1 & 2 & 1 & 2 & 1 \\
\hline 18 & 6 & -2 & 6 & 1 & 6 & 1 & 2 & 1 & 2 & 1 & 2 & 1 \\
\hline 19 & 7 & -2 & 7 & 1 & 6 & 0 & 2 & 1 & 2 & 1 & 2 & 1 \\
\hline 20 & 7 & -2 & 7 & 1 & 7 & 1 & 1 & -1 & 2 & 0 & 2 & 0 \\
\hline 21 & 9 & -1 & 8 & 2 & 7 & 1 & 2 & 0 & 2 & 0 & 3 & 1 \\
\hline 22 & 9 & -1 & 8 & 1 & 8 & 1 & 2 & 0 & 3 & 1 & 3 & 1 \\
\hline 23 & 10 & 0 & 8 & 1 & 8 & 1 & 2 & 0 & 3 & 1 & 3 & 1 \\
\hline 24 & 12 & 1 & 9 & 2 & 9 & 1 & 3 & 1 & 3 & 1 & 3 & 1 \\
\hline 25 & 15 & 4 & 9 & 1 & 9 & 1 & 3 & 1 & 3 & 1 & 4 & 2 \\
\hline 26 & 16 & 4 & 9 & 1 & 10 & 2 & 3 & 0 & 4 & 1 & 4 & 1 \\
\hline 27 & 17 & 5 & 9 & 1 & 11 & 2 & 3 & 0 & 4 & 1 & 4 & 1 \\
\hline 28 & 17 & 4 & 9 & 0 & 12 & 3 & 4 & 1 & 5 & 2 & 5 & 2 \\
\hline 29 & 18 & 5 & 11 & 2 & 12 & 2 & 4 & 0 & 6 & 2 & 5 & 1 \\
\hline 30 & 30 & 16 & 11 & 1 & 13 & 2 & 6 & 2 & 6 & 2 & 6 & 2 \\
\hline 31 & . & . & 11 & 1 & 14 & 3 & 6 & 1 & 7 & 2 & 6 & 1 \\
\hline 32 & . & . & 11 & 0 & 15 & 3 & 7 & 2 & 7 & 2 & 7 & 2 \\
\hline 33 & . & . & 12 & 1 & 15 & 2 & 8 & 2 & 9 & 3 & 8 & 2 \\
\hline 34 & . & . & 14 & 2 & 16 & 3 & 8 & 1 & 10 & 3 & 11 & 4 \\
\hline 35 & . & . & 14 & 1 & 16 & 2 & 9 & 1 & 11 & 3 & 11 & 3 \\
\hline 36 & . & . & 15 & 1 & 17 & 2 & 9 & 0 & 11 & 2 & 12 & 3 \\
\hline 37 & . & . & 15 & 0 & 18 & 2 & 19 & 8 & 16 & 5 & 14 & 3 \\
\hline 38 & . & . & 15 & -2 & 19 & 1 & 19 & 5 & 17 & 3 & 18 & 4 \\
\hline 39 & . & . & 19 & -1 & 20 & 0 & 20 & 0 & 20 & 0 & 21 & 1 \\
\hline
\end{tabular}


Note. Italics indicate the threshold rank difference: Empirical best response - Equilibrium. Differences that are at least four in absolute value are highlighted in gray. No observed searches in the minority rule condition $(q=1)$ in Experiment 1 went beyond alternative 30, so that we could not estimate continuation expected payoff beyond that. We assumed an empirical best response threshold of 30 for that alternative.

\section{A.8. Analysis of Post-Experimental Questionnaire Data in Experiment 2}

On average, subjects in the minority rule condition scored 3.7 questions correct out of 5 (s.d. $=0.7$ with committee as the unit of analysis) in the post-experimental questionnaire, in other words a $74 \%$ average rate of correctly answering a question. The corresponding averages for the majority and unanimity rule conditions were $3.8($ s.d. $=0.7)$ and $4.0($ s.d. $=0.6)$ out of 5 , respectively. There was no significant difference in these average scores across voting rule conditions. A three-condition between-subjects ANOVA for the average scores, with condition as the single independent variable and committee as the unit of analysis, yielded $F(2,39)=0.94, p>0.4$.

We also examined whether exposure to the decision task, in terms of the total number of searches done, would impact the questionnaire scores. In the minority and majority rule conditions a committee's average number of searches per game did not have a significant correlation with the average score of the committee in the questionnaire $(p>0.1)$. Meanwhile, the correlation was significantly positive $(=0.38 ; p$ $<0.05)$ in the unanimity rule condition. This finding suggests that some of the especially long searches in

the unanimity rule condition (cf. Table 3, as well as Online Appendix A.5) did create some differences among committees within that condition regarding understanding of the task; however, the effect was not sufficiently impactful to lead to a significantly higher score in that condition compared with the other two.

Hence, we conclude that subjects' understanding of the decision task was generally and uniformly high across conditions; in addition, such level of understanding was achieved early in the sessions. 


\section{A.9. Analysis Regarding Hypothesis Testing and Familywise Errors}

Regarding false positives issues in hypothesis testing, we gauge the reliability of our claims using Maniadis et al. (2014)'s approach, and focusing on the main findings of under- or over-search across conditions in the three experiments. There are two major dependent variables (MSL and MAR) and seven experimental conditions in our three experiments, which form a total of 14 means. For 13 of these means, we claim deviations from equilibrium predictions at significance level $p<0.05$ according to $t$-tests (see Table 3 ). Using the observed means as proxy of the true means, we find that the powers of these significant deviations range from 0.6 to effectively 1 . We then calculate the post-study probability (PSP) under a range of scenarios following the approach of Maniadis et al. (2014). Specifically, we use representative values of powers at 0.6 and 0.9 , priors at $0.05,0.1$, and 0.55 , and assume that there are no competing studies. The PSP estimates thus obtained are listed in table (a) below. They show that even with priors as low as 0.1 , our PSP estimates are at least 0.5 (see Maniadis et al. 2014 on interpreting PSPs).

(a) Post-Study Probability (see Maniadis et al. 2014) Estimates for the Experiments

\begin{tabular}{c|cc}
\hline & \multicolumn{2}{|c}{ Power } \\
Prior & 0.6 & 0.9 \\
\hline 0.01 & 0.11 & 0.15 \\
0.1 & $\mathbf{0 . 5 7}$ & $\mathbf{0 . 6 7}$ \\
0.55 & $\mathbf{0 . 9 4}$ & $\mathbf{0 . 9 6}$ \\
\hline
\end{tabular}

Note. Estimates above 0.5 are highlighted in bold.

Regarding familywise errors, our approach is to employ Holm-Bonferroni correction. The results are listed in table (b) below. Overall, the deviations remain significant at $p<0.05$ after the corrections; three of the deviations are significant at unadjusted $p<0.01$ but only significant at Holm-Bonferroni adjusted $p<0.05$. 
(b) Holm-Bonferroni Corrections for $p$-values of Significant Deviations from Equilibrium

\begin{tabular}{|c|c|c|c|c|}
\hline \multirow[b]{2}{*}{ Experiment } & \multirow[b]{2}{*}{ Voting rule condition } & \multirow[b]{2}{*}{ Dependent variable } & \multicolumn{2}{|c|}{$p$-value } \\
\hline & & & Unadjusted & Holm-Bonferroni \\
\hline 1 & 1 & MAR & 0.0053 & 0.0265 \\
\hline 1 & 1 & MSL & 0.0002 & 0.002 \\
\hline 1 & 2 & MAR & 0.0173 & 0.0346 \\
\hline 1 & 2 & MSL & 0.0028 & 0.0168 \\
\hline 1 & 3 & MAR & $<0.0001$ & $<0.0013$ \\
\hline 1 & 3 & MSL & 0.0101 & 0.0303 \\
\hline 2 & 1 & MAR & 0.0002 & 0.0018 \\
\hline 2 & 1 & MSL & $<0.0001$ & $<0.0013$ \\
\hline 2 & 2 & MAR & 0.0386 & 0.0386 \\
\hline 2 & 2 & MSL & $<0.0001$ & $<0.0013$ \\
\hline 2 & 3 & MAR & 0.0007 & 0.0049 \\
\hline 3 & - & MAR & 0.0005 & 0.004 \\
\hline 3 & -- & MSL & 0.0073 & 0.0292 \\
\hline
\end{tabular}

Note. Bold $p$-values indicate deviations that are significant at unadjusted $p<0.01$ but at Holm-Bonferroni adjusted $p<0.05$. 


\section{A.10. Optimal Voting Rules $q^{*}$ under Equilibrium for a Sample of Committee Sizes $m$ and Preference} Correlations $\mu(n=40)$

\begin{tabular}{c|ccc|ccc|ccc|c}
\hline & \multicolumn{3}{|c|}{$\mu=0$} & \multicolumn{3}{|c|}{$\mu=1 / 3$} & \multicolumn{3}{|c|}{$\mu=2 / 3$} & $\mu=1$ \\
$m$ & $q^{*}$ & $q^{* / m}$ & MAR & $q^{*}$ & $q^{* / m}$ & MAR & $q^{*}$ & $q^{* / m}$ & MAR & MAR \\
\hline 1 & 1 & 1.00 & 3.3 & 1 & 1.00 & 3.3 & 1 & 1.00 & 3.3 & 3.3 \\
2 & 2 & 1.00 & 7.3 & 2 & 1.00 & 5.1 & 2 & 1.00 & 4.0 & 3.3 \\
3 & 3 & 1.00 & 10.9 & 3 & 1.00 & 6.4 & 3 & 1.00 & 4.4 & 3.3 \\
4 & 3 & 0.75 & 11.8 & 4 & 1.00 & 7.6 & 4 & 1.00 & 4.8 & 3.3 \\
5 & 4 & 0.80 & 12.5 & 4 & 0.80 & 8.6 & 5 & 1.00 & 5.2 & 3.3 \\
6 & 5 & 0.83 & 14.1 & 5 & 0.83 & 8.5 & 6 & 1.00 & 5.6 & 3.3 \\
7 & 5 & 0.71 & 14.5 & 6 & 0.86 & 8.7 & 6 & 0.86 & 6.0 & 3.3 \\
8 & 6 & 0.75 & 14.8 & 7 & 0.88 & 9.1 & 7 & 0.88 & 5.8 & 3.3 \\
9 & 6 & 0.67 & 15.4 & 7 & 0.78 & 9.5 & 8 & 0.89 & 6.0 & 3.3 \\
10 & 7 & 0.70 & 15.4 & 8 & 0.80 & 9.5 & 9 & 0.90 & 6.0 & 3.3 \\
20 & 13 & 0.65 & 17.0 & 16 & 0.80 & 10.8 & 17 & 0.85 & 6.7 & 3.3 \\
50 & 30 & 0.60 & 18.4 & 38 & 0.76 & 11.7 & 43 & 0.86 & 7.3 & 3.3 \\
100 & 57 & 0.57 & 19.0 & 74 & 0.74 & 12.2 & 85 & 0.85 & 7.5 & 3.3 \\
\hline
\end{tabular}

Note. A voting rule with $q^{*}$ means a threshold number of $q^{*}$ votes are required to stop the search. When $\mu$ $=1$ (perfectly correlated preferences), all the committee members follow the same benchmark strategy and always accept/reject alternatives unanimously, so that all voting rules are equally optimal. Moreover, the optimized mean absolute rank is the same $(=3.3)$ regardless of group size. The latter values are included in the table for ease of comparison. Likewise, the trivial case of $m=1$ is included for comparison's sake. 